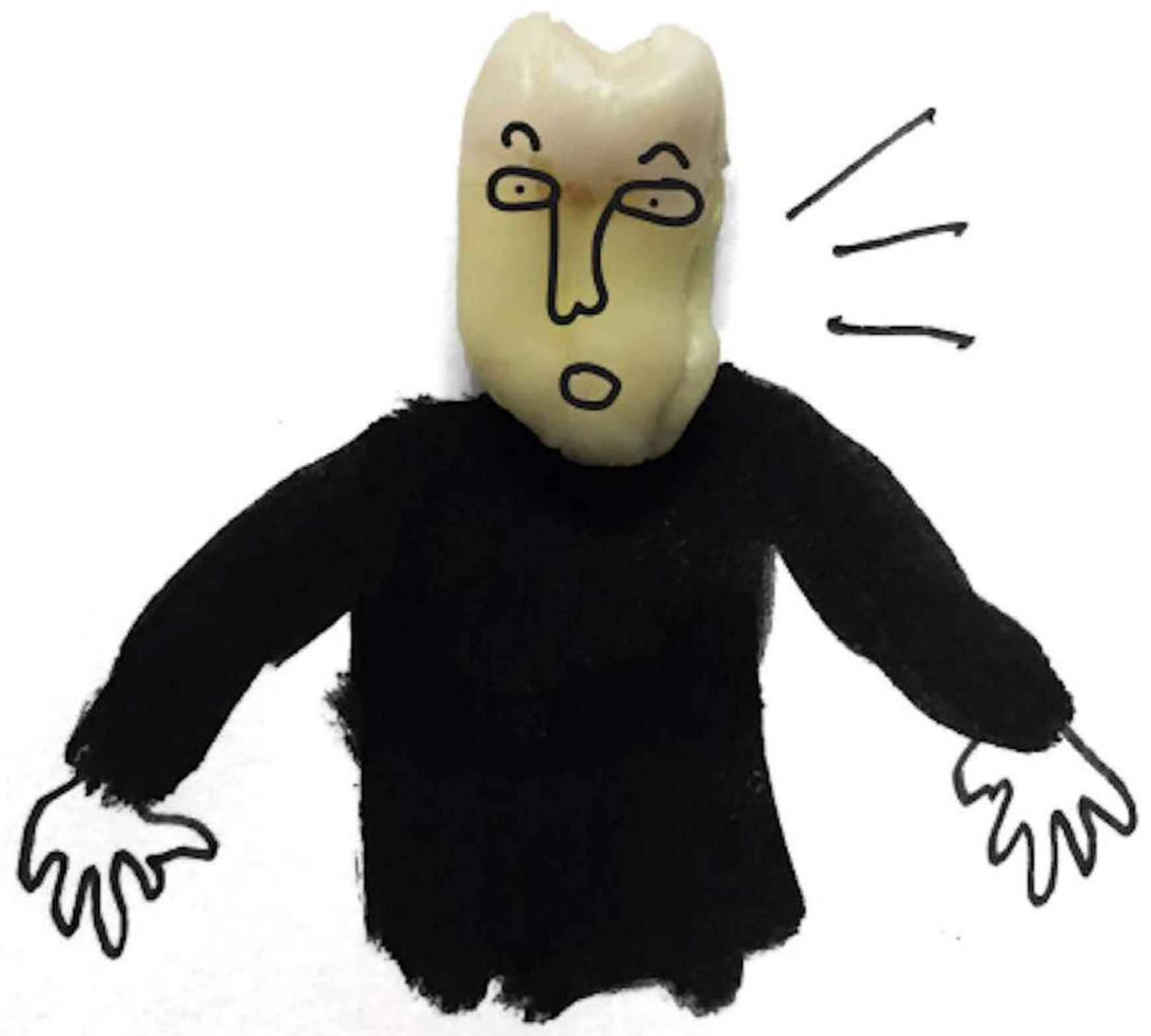


$\mathrm{D}+\mathrm{E}$ :

fundamentos,

problemática

y soluciones

pedagógicas

Jorge Hernán Maya Castaño 


\section{D+E: fundamentos, problemática y soluciones pedagógicas}

\section{Jorge Hernán Maya Castaño}

jmayacas@eafit.edu.co | Doctor en Ingeniería Mecánica, Especialidad de Diseño de Productos, Universidad de Tecnología de Compiègne, Francia. Profesor e investigador, Laboratorio GRID, Universidad EAFIT, Medellín, Colombia.

Fecha de recepción: 23 marzo 2017

Fecha de aceptación: 19 julio 2017

\section{Resumen}

En mercados saturados, el diseño para las emociones, $D+E$, es una alternativa para ofrecer beneficios al usuario que otros productos competidores no presentan. No obstante, el D+E basado sólo en la intuición puede derivar en soluciones estereotipadas que serán desaten-didas en el mercado o que no generarán en el usuario las emociones deseadas por el diseñador o empresa. Este artículo aborda los principales problemas que implica la pedago-gía de los métodos del D+E y ofrece fundamentos, clarificando qué son las emociones, cuáles son sus componentes y cómo se diferencian de otros sentimientos afectivos y mos-trando los mecanismos que explican su estructura y formación. Se presenta una variedad de métodos del $D+E$, desde baja hasta altamente estructurados, y se examina cómo se adaptan a diversas condiciones de los alumnos y de las empresas. Se presentan ejemplos resultantes de dichos métodos. Finalmente, se discuten los problemas disciplinares y epis-temológicos del campo.

Palabras clave | diseño para las emociones; D+E; pedagogía del diseño; emociones; experiencia de usuario; diseño centrado en el usuario. 
D+E: FUNDAMENTOS, PROBLEMÁTICA

Y SOLUCIONES PEDAGÓGICAS

\begin{abstract}
In saturated markets, the design for emotions, $D+E$, is an alternative to offer benefits to the user that other competing products cannot deliver. However, D+E based only on intuition can lead to stereotyped solutions that will be neglected in the market or that will not generate in the user the emotions desired by the designer or company. This article addresses the main problems involved in the pedagogy of $D+E$ 's methods and provides a basis for clarifying what emotions and their components are, and how they differ from other affective feelings and showing the mechanisms that explain their structure and formation. A variety of $D+E$ methods are presented, ranging from low to highly structured, and it is examined how they adapt to different students and companies conditions. Examples resulting from such methods are presented. Finally, the disciplinary and epistemological problems of the field are discussed.
\end{abstract}

Keywords | design for emotion; D+E; design pedagogy; emotions; user experience; UCD. 
D+E: FUNDAMENTOS, PROBLEMÁTICA

Y SOLUCIONES PEDAGÓGICAS

\section{El desafío pedagógico del diseño para las emociones}

El diseño para las emociones o $D+E$, es un campo de conocimiento que se encuentra en desarrollo y en el que aún no hay paradigmas dominantes (Blythe, Overbeeke, Monk, \& Wright, 2004; Desmet, van Erp, \& Karlsson, 2008; Van Gorp \& Adams, 2012; Norman, 2007). Por ende, su enseñanza-aprendizaje es un gran desafío, tanto para el docente que debe identificar los contenidos y las estrategias para que haya un aprendizaje eficaz (Merrill, D., Drake, L., Lacy, M., Pratt, J. 1996), como para los alumnos que deberán tratar de entender, de primera mano, cómo funcionan en ellos mismos muchos fenómenos afectivos que sienten cotidianamente. Además, dicho campo de conocimiento posee vacíos explicativos que tanto el docente como los alumnos deberán identificar y aceptar. A pesar de esto, algunas teorías de las emociones han dado lugar a conceptos y métodos en los cuales apoyarse al hacer el D+E.

En este artículo se muestra el caso de la enseñanza-aprendizaje del diseño para las emociones en el curso Diseño para las emociones ofrecido simultáneamente para alumnos de último año en ingeniería y del postgrado en rediseño de productos. El curso se ha ofrecido en once ocasiones, durante seis años, en la facultad de ingeniería de la Universidad EAFIT, en Medellín, Colombia. Quien asista al curso de diseño para las emociones deberá entender la doble condición que habita alguien que desea aprender en la educación formal: es estudiante, quien se presenta con la disposición de adquirir conocimientos y habilidades, y es aprendiz, ya que debe encontrar sentido y cambiar su comportamiento basado en sus experiencias (Merrill et al., 1996). Como sostiene el filósofo e investigador de la educación José Antonio Marina "la profesión de los docentes no es enseñar; la profesión de los docentes es a ver cómo nos las ingeniamos para que nuestros alumnos aprendan, porque eso es lo importante" (Marina, J. A. 2015). Es por esto que se mostrará a lo largo de la presentación ejemplos de proyectos realizados por los alumnos del curso, que enseñan el potencial de aplicación de lo aquí expuesto y que, se piensa, dan indicios de cuánto puede aprenderse presentando estos conceptos y herramientas del D+E en forma estructurada. 
D+E: FUNDAMENTOS, PROBLEMÁTICA

Y SOLUCIONES PEDAGÓGICAS

El programa de curso aquí expuesto fue creado siguiendo los pasos expuestos por el método ADDIE (Jones, 2014) que propone un método de diseño instruccional consistente en las siguientes etapas: análisis, diseño, desarrollo, implementación y evaluación. A grosso modo, en la fase de análisis se establecen los objetivos en función del público; en la de diseño se definen los temas, las herramientas de evaluación, la planificación y los recursos necesarios; en la fase de desarrollo se usa la información ya recabada para plantear el programa del curso de manera tal que se pueda ejecutar el curso. En la implementación, se modifica sucesivamente el programa de manera a lograr una máxima eficiencia en el aprendizaje y los resultados emanados del curso. Este proceso se ha repetido entonces durante la historia del curso. En la evaluación, el proyecto del curso es testeado para examinar qué, cómo, por qué y cuándo se lograron o no los objetivos del curso. En el artículo se discutirán y presentarán entonces los conceptos que son problemáticos para los estudiantes y cómo se han solucionado. En detalle, se explicarán las herramientas que han funcionado de forma exitosa, acompañadas por ejemplos de su aplicación.

El artículo está estructurado en cuatro secciones. En lo que resta de la primera se presenta el marco teórico que sustenta el curso y que constituye su mensaje de fondo; además se explica cómo se puede hacer el $D+E$, intuitivamente o con métodos. En la sección segunda se presentan los diferentes problemas pedagógicos que se han enfrentado en el desarrollo del curso. En la tercera se explican los diversos métodos presentados y/o aplicados, acompañados de ejemplos. En la cuarta se presentan las conclusiones y perspectivas.

\section{Qué es el diseño para las emociones}

y la experiencia del usuario con el producto, UX

\section{El diseño y las necesidades del usuario con el producto}

En términos generales el diseño de productos consiste en responder a unas necesidades del usuario a través de ciertos servicios que brinda el producto; estos servicios pueden configurarse manipulando sus características físicas (Vogel \& Cagan, 2001). Jordan (1998; 
D+E: FUNDAMENTOS, PROBLEMÁTICA

Y SOLUCIONES PEDAGÓGICAS

2002) reconoce tres tipos de necesidades de los usuarios con el producto. Las dos primeras, cubiertas por la funcionalidad y la usabilidad del producto serían necesidades básicas que soportan las terceras: las necesidades de obtención de placer con el producto. Por ejemplo, en estas últimas encontraríamos: necesito un secador de manos que genere un chorro fuerte de aire a una temperatura agradable, y que no me haga rabiar debido a su lento funcionamiento. Estas últimas necesidades son de tipo psicológico-afectivo, es decir que conciernen la experiencia del usuario con el producto, o UX.

\section{Lo emocional, semántico y estético como niveles}

\section{de la experiencia del usuario con el producto}

Existen muchas definiciones diferentes de qué es la UX y de cuáles son sus componentes o niveles (Ariza \& Maya, 2014; Hekkert \& Schifferstein, 2008 ; Hassenzahl \& Tractinsky, 2006; Ortíz-Nicolás, \& Aurisicchio, 2011; Abbasi et al., 2012; Karapanos, 2013; Law, Vermeeren, Hassenzahl, \& Blythe, 2007). Aquí adoptaremos la de Hekkert y Schifferstein (2008) debido a su simpleza y alto poder analítico. La UX es la autoconsciencia que tiene el usuario de los efectos psicológicos sentidos con el producto (Hekkert \& Schifferstein, 2008; Schifferstein \& Hekkert, 2008). La UX es por definición subjetiva y está regida por el núcleo afectivo. Dicho núcleo (core affect), está conformado por dos dimensiones: la valencia (positiva o negativa; placer o displacer) y el nivel de intensidad (arousal en inglés), que puede ser alto o bajo.

La UX presenta entonces tres niveles: emocional, estético y semántico (Hekkert \& Schifferstein, 2008; Schifferstein \& Hekkert, 2008). El emocional se refiere a todos los fenómenos que conciernen a las emociones, sentimientos y estados de ánimo (Desmet en: Schifferstein \& Hekkert, 2008). El estético se refiere al placer (o displacer) que podemos lograr a través de la estimulación de nuestros sentidos (Hekkert \& Leder, 2008; Van Rompay, T., Hekkert, P., Saakes, D., \& Russo, B., (2005). El semántico se refiere a los diferentes tipos de significados comunicados por el producto: símbolos, denotaciones, connotaciones, características expresivas de los productos y significados corporificados (Krippendorff \& Butter, 1984; van Rompay et al., 2005). 
D+E: FUNDAMENTOS, PROBLEMÁTICA

Y SOLUCIONES PEDAGÓGICAS

Uno de los problemas que debe enfrentar la enseñanza del D+E es entonces el carácter afectivo de la UX, ya que las emociones, el sentimiento estético y los significados son solo algunos de los aspectos psicológicos que son regidos por nuestro sistema afectivo. La actitud, hacia un producto o hacia su marca, o nuestra personalidad y preferencias también son fenómenos afectivos, entre otros aspectos más (Scherer, 2005).

En el campo del diseño para la UX se diferencia entre diseñar la UX y diseñar para la UX (Hekkert \& Schifferstein, 2008). De igual forma, en el "diseño de las emociones" se asumirían las emociones como un fenómeno determinable, el cual es posible anticipar y controlar a través del diseño. En el "diseño para las emociones", $D+E$, estas se asumen como un fenómeno complejo, con lo que el diseñador, a través de su diseño, solo puede aportar elementos que propicien una emoción buscada, pero sin garantizar que se vaya a presentar.

¿Cuál es entonces la importancia del D+E hoy? La globalización ha tenido como efecto la apertura de los mercados con lo que muchas categorías de productos presentan unos niveles de funcionalidad y de usabilidad similares (Matzler \& Hinterhuber, 1998). Pensemos por ejemplo en los electrodomésticos de gama básica: refrigeradores y hornos microondas cumplen muy bien su propósito funcional, presentando también una muy buena usabilidad. Es por esto que es en las necesidades de tipo psicológico, referentes a la UX, en donde se presenta la posibilidad de competir y/o diferenciarse fácilmente (Matzler \& Hinterhuber, 1998). El modelo japonés de la calidad de Kano, ya advertía esto desde los años 80s (Matzler \& Hinterhuber, 1998).

\section{Cómo se hace el diseño para las emociones}

El diseño para la UX, como muchos aspectos del diseño basado en el usuario puede realizarse utilizando métodos que se presentan sobre un continuo que incluye, en un extremo, métodos poco estructurados, utilizando únicamente la intuición del diseñador, hasta el otro extremo, en donde existen métodos altamente estructurados tales como la ingeniería Kansei, KE. En medio se encontrarían los métodos semi-estructurados, que poseen algunas 
D+E: FUNDAMENTOS, PROBLEMÁTICA

Y SOLUCIONES PEDAGÓGICAS

de sus instrucciones estructuradas, pero otras no (Roozenburg \& Eekels, 1995). A continuación hacemos un recorrido de métodos y herramientas para el $D+E$, partiendo desde los más intuitivos y de baja estructura, hasta los altamente estructurados con instrucciones bien definidas.

\section{La intuición como método para el D+E}

y los aspectos semánticos y estéticos de la experiencia

Históricamente, la intuición ha sido el método utilizado por los diseñadores para atacar el D+E y la UX. Además, la utilización de métodos por los diseñadores industriales y de productos en la industria ha mostrado siempre un predominio del uso de la intuición (Fujita \& Matsuo, 2005; Wright, Campello, Segre, Benedetto-Neto, \& Araujo, 1995) y una inmediatez en los procesos de toma de decisión. Presentar una idea o concepto a los compañeros del departamento de diseño, para obtener insights que orienten una toma de decisiones de base intuitiva, es una práctica extensamente reportada (Vermeeren, A., Law, E. L. -C., Roto, V., Obrist, M., Hoonhout, J. \& Väänänen-Vainio-Mattila. K. (2010); Wright, I., Campello, A., Segre, F., Benedetto-Neto, H. \& Araujo, C. (1995).

En las ciencias cognitivas, la intuición es definida como una manera de procesar la información de forma automática, basándose en estándares personales y afectivos (Gigerenzer \& Todd, 1999; Tonetto \& Tamminen, 2015). Muchas veces los diseñadores toman decisiones de forma intuitiva consideranco el diseño para la UX, tratando de inferir cómo desencadenar una experiencia particular en el usuario (Tonetto \& Tamminen, 2015).

Los diseñadores diseñan para las emociones basándose en las capacidades de inferir, imaginar y predecir las emociones y otros estados afectivos en los demás, tal como lo explica la teoría de la mente (Theory of Mind o ToM) en ciencias cognitivas (Byom \& Mutlu, 2013). Esto se hace de dos formas. Primero, leyendo algunas de las señales comportamentales asociadas, sobre todo, las expresiones del rostro, gestos corporales e inflexiones de la voz (Byom \& Mutlu, 2013). Sin embargo, los humanos no son capaces de realizar esto de forma sistemática, con lo que frecuentemente se equivocan en tales lecturas. Esto hace que estas 
D+E: FUNDAMENTOS, PROBLEMÁTICA

Y SOLUCIONES PEDAGÓGICAS

capacidades sean poco confiables y susceptibles al error. Segundo, el diseñador se puede apoyar en su propia introspección. La introspección, aún con un entrenamiento deliberado ha mostrado ser poco confiable como método: reportes introspectivos de diferentes laboratorios, sobre las mismas experiencias, dan resultados distintos (Schwitzgebel, 2016). Además, lo que se alcanza a percibir en la propia mente es la punta del iceberg de la conciencia propia, con lo que la mayoría de los fenómenos afectivos quedan lejos del alcance de la introspección (Eagleman, 2011).

El uso de la intuición, secundado por las habilidades conferidas por la teoría de la mente y la introspección pueden ser útiles para el $\mathrm{D}+\mathrm{E}$, sin embargo, son métodos poco confiables, sobre todo entre los diseñadores de bajo nivel de experiencia. En otras palabras, el problema es que el diseñador experto puede poseer una buena intuición pero no el inexperto (Myers, 2004); es allí entonces en donde las herramientas más estructuradas deben guiar y apoyar el proceso de diseño y de toma de decisiones. De todas formas, en un curso de D+E se debe tratar de complementar la intuición del diseñador y no necesariamente reemplazarla.

\section{Los heurísticos de diseño}

Los heurísticos son reglas simples de uso frecuente en diseño, expresadas en lenguaje corriente y procedentes de la experiencia, cuya aplicación aproxima al diseñador a una solución exitosa, pero sin garantizarla (Yilmaz, 2010). Diferentes estudios han reportado un número importante de heurísticos que conciernen la usabilidad, funcionalidad y arquitectura del producto, pero un número muy bajo se refieren al D+E o más ampliamente al diseño para la UX (Calle-Escobar, Mejía-Gutiérrez, Nadeau, \& Pailhes, 2014; Yilmaz, 2010; Daly, Christian, Seifert, Gonzalez, \& Yilmaz, 2014). Es probable que muchos heurísticos de diseño de la UX permanezcan tácitos dentro del proceso de diseño y que no hayan sido identificados, sencillamente porque los investigadores estaban a la búsqueda de heurísticos de corte cognitivo, en un enfoque del diseño como solución de problemas (ver: Yilmaz 2010). Los heurísticos ligados al D+E y la UX requerirían herramientas diferentes para su extracción (Cadavid, Ruiz \& Maya, 2016). 
D+E: FUNDAMENTOS, PROBLEMÁTICA

Y SOLUCIONES PEDAGÓGICAS

\section{Métodos semiestructurados}

Los métodos semiestructurados combinan algunas acciones que se encuentran claramente definidas con otras que no lo son (Roozenburg \& Eekels, 1995). Por ejemplo, "realice una escala multidimensional para identificar las emociones que reportan usuarios frente a una muestra de productos" es claramente definida; "desarrolle tres alternativas del producto", no lo es. Desmet, Porcelijn, y Van Dijk (2007) exponen un método semiestructurado para el D+E que forman el wow (definido como mezcla de fascinación, sorpresa agradable y deseo), en una aplicación al diseño de un celular. En este, se encuesta a treinta y cinco usuarios mediante el uso de PrEmo, para que juzguen un conjunto de ocho celulares según diez emociones, cinco de emociones placenteras y cinco displacenteras, obteniendo un índice del Wow. PrEmo es un método de medición de emociones en los productos desarrollado por Desmet (2007). Enseguida los datos son analizados usando análisis de correspondencias y como resultado se identifican y representan sobre un mapa perceptual los celulares que son más cercanos entre sí en términos de las tres emociones de interés. Luego, a través de un proceso de diseño más o menos complejo, se da especial énfasis al desarrollo de la arquitectura del producto. Se busca que el celular presente tres capas de interacción (como cámara, como celular y para navegación en internet). Se llega finalmente a una propuesta que será prototipada y luego evaluada en las mismas condiciones del estudio inicial. Dicha propuesta resulta ser la más susceptible de producir wow, separada por una leve diferencia frente al segundo mejor celular.

\section{Métodos estructurados}

Estos métodos ofrecen unas instrucciones claramente definidas y un orden en el que deben ejecutarse (Roozenburg \& Eekels, 1995). Frecuentemente su parte cuantitativa es muy importante y la subjetividad de quien aplica el método está reducida a una mínima expresión. Cuando el nivel de estructura es máximo y sus instrucciones se encuentran en un lenguaje formal, se habla de un método algorítmico que siempre dará resultados consistentes en el tiempo (Roozenburg \& Eekels, 1995). La ingeniería Kansei (KE), es un ejemplo de método estructurado para el $D+E$. Kansei es una palabra japonesa que se aproxima en significado a lo definido aquí para la UX (Nagamachi, 2010). La KE se empezó a desarrollar 
D+E: FUNDAMENTOS, PROBLEMÁTICA

Y SOLUCIONES PEDAGÓGICAS

desde los años 70s para ser aplicada a proyectos de diseño de productos que implicaran alto riesgo financiero (Nagamachi, 2010). Para una presentación completa de la KE, (ver Nagamachi, 2010).

En tanto que ingeniería, la aplicación de la KE es capaz de garantizar sus resultados con una alta precisión (Nagamachi, 2002). La contraparte de esto es, en lo metodológico, el exigir muestras de población muy grandes para realizar sus estudios, el tener que implementar técnicas estadístico-matemáticas relativamente sofisticadas para analizar los datos obtenidos y poder vincular las palabras Kansei con características del producto deseado y el tener que dirigir sus productos a un segmento de población claramente caracterizado (Lokman, 2010; Nagamachi, 2010; Schütte et al., 2004). En conclusión, la KE es un método altamente preciso para el $\mathrm{D}+\mathrm{E}$, pero es demasiado robusto frente a las capacidades de las PYMES, haciendo muy costosa su implementación.

Habiendo explicado el nivel de estructuración de métodos para el $D+E$, se presentan enseguida aquellos ofrecidos en el curso.

\section{Desarrollo del curso de D+E. Problemas y soluciones inherentes a los fundamentos del D+E.}

La discusión de los métodos del D+E presentados se hace en cuatro grandes secciones. Primero, se discute cómo diferenciar las emociones de los otros dos niveles de la UX. Segundo, se explica cómo se han identificado los componentes de la emoción en el curso y cuál es el interés de hacerlo. Tercero, se muestra cómo los circunflejos de emociones son un modelo que explica su orden. Cuarto, se presentan los problemas disciplinares y epistemológicos vinculados al curso de D+E presentado.

A continuación presentamos dichos conceptos básicos del $D+E$, pero haciendo énfasis en los problemas pedagógicos encontrados y en la manera cómo se han resuelto. 


\section{Primera sección: ¿Cómo se diferencian entre sí los niveles de la UX?}

Desde el inicio del curso este aspecto resulta fundamental ya que si se va a tratar con emociones, lo normal es que las personas confundan éstas con otros fenómenos afectivos y con los otros dos niveles de la UX, el estético y el semántico. Dos ejemplos: primero, la admiración, el optimismo y la confianza, aunque aparecen referidas como emociones relevantes con los productos (Ortíz- Nicolás \& Hernández-López, 2008) también son valores, al menos en español. Segundo, el término "armonía" es usado a la vez como un sentimiento emocional, un valor o un determinante estético.

Hay que enseñar entonces a los alumnos a discriminar lo que están sintiendo, de acuerdo con las definiciones de cada uno de los niveles. Una de las maneras de discriminar estos sentimientos es mostrando estímulos que les generen una respuesta de alta intensidad. Por ejemplo, imágenes de personas que hayan sufrido accidentes, Figura 1, son de utilidad para suscitar en el alumno una reacción emocional intensa y así poderla identificar introspectivamente.

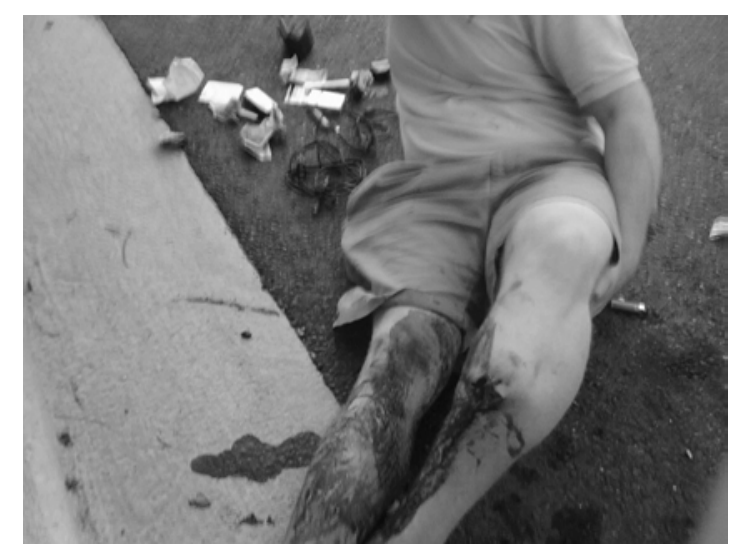

Figura 1. Chad fitz, 2008. Picture of broken leg. La foto original, en colores, muestra una persona accidentada con una fractura expuesta de tibia. La foto genera una reacción emocional intensa en quienes la ven. commons.wikimedia.org/wiki/File:Broken_leg.jpg. 
D+E: FUNDAMENTOS, PROBLEMÁTICA

Y SOLUCIONES PEDAGÓGICAS

\section{Diferenciar lo emocional de lo estético y lo semántico}

Es importante diferenciar la emoción, sentimiento subjetivo inmediato al estímulo que la causó y que posee reacciones viscerales asociadas, del sentimiento, que es el tipo de sentimiento afectivo más estable, posterior a la emoción y que es expresado de manera comportamental: a través del lenguaje o gestos o expresiones del rostro. El sentimiento se puede falsear, no así la emoción.

Mostrar las diferencias con el sentimiento estético puede ser un poco más difícil ya que debe hacerse a través de imágenes. Una manera de hacerlo es a través de ejemplos de productos que posean una gran riqueza estética, por ejemplo, los automóviles Ferrari, Figura 2. Se debe explicar qué son las formas, colores, el manejo de materiales y proporciones de estos autos, es decir, su impacto sobre los sentidos perceptivos, lo que genera dicho sentimiento. Este sentimiento se presenta, a diferencia de las emociones, de manera automática, sin que haya un interés (concern) básico (Hekkert \& Leder, 2008). En otros términos, puede ocurrir que a una persona no le interesen los automóviles deportivos o la marca Ferrari, sin embargo, tendrá un sentimiento estético, pudiendo, eso sí, variar en intensidad entre personas.

Otro elemento de confusión en los alumnos viene de la presencia de sentimientos emocionales posteriores a la generación del sentimiento estético. Hekkert y Leder (2018) explican claramente que esos sentimientos emocionales, y significados semánticos además, pueden presentarse posteriormente al surgimiento del sentimiento estético. Es claro, sin embargo, que por el momento, la secuencia de procesamiento de la información allí no se entiende muy bien (Leder et al., 2004). Similarmente, se sabe que variables de índole cognitiva, tales como la familiaridad o tipicidad, pueden estar a la base del sentimiento estético (Hekkert, 1995; Hekkert, Thurgood, \& Whitfield, 2013). Esto significa que el automóvil Ferrari que es enseñado, generaría un sentimiento estético positivo debido a que para un individuo representa un cierto grado de familiaridad (Hekkert, 1995). En este sentido, un automóvil que fuese completamente extraño al individuo no le generaría dicho sentimiento agradable o le generaría un sentimiento estético de valencia negativa. Esto supone un pre procesamiento semántico (cognitivo) de la información, con lo que la automaticidad del sentimiento estético se vería comprometida (Leder, Belke, Oeberst, \& Augustin, 2004). 
D+E: FUNDAMENTOS, PROBLEMÁTICA

Y SOLUCIONES PEDAGÓGICAS

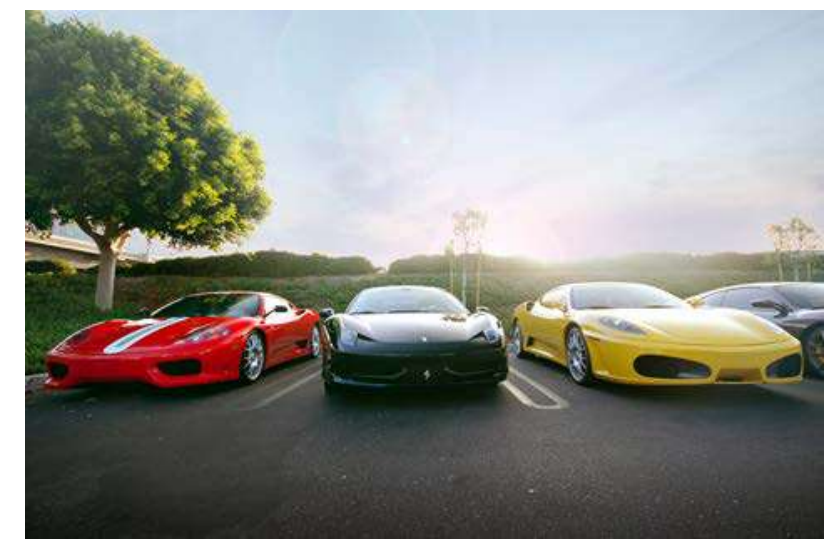

Figura 2. Automóviles Ferrari de diferentes modelos, similares

a los mostrados como estímulo para identificar el sentimiento estético. Fuente: Axion 23. Licencia Creative Commons 2.0

upload.wikimedia.org/wikipedia/commons/6/61/360_Challenge\%2C_458_ Italia_Spider._F430_\%288560770046\%29.jpg?uselang=es

\section{Diferenciar lo semántico de los estético y lo emocional}

Para diferenciar lo semántico de lo estético y emocional se acude a explicar los diferentes tipos de elementos semánticos que se encuentran comúnmente en diseño de productos. Esto se hace a través de preguntar a los alumnos el tipo de significados que les transmite un automóvil Mercedes que se muestra en una fotografía acompañada del texto ¿Qué significados transmite este producto? Se les invita a diferenciar, por ejemplo, símbolos, de valores (de la marca) o de características expresivas del producto (es "para adultos maduros"). Se diferencia el significado particular, del tipo de elemento semántico y de su significante. Por ejemplo un significado particular es "vehículo de alto estatus", lo cual es un valor de la marca Mercedes (tipo de elemento semántico) cuyos significantes se encuentran en la publicidad de la marca y en la cultura popular sobre dicha marca. Otro ejemplo puede ser el significado "elegante", el cual es un valor de marca, cuyo significante puede ubicarse en la ausencia de detalles formales superfluos en la carrocería y en sus proporciones. Desde Osgood \& Suci $(1955 ; 1962)$ se ha demostrado que unos espacios semánticos podían mostrarse como conceptos sobre un continuo definible por un par de términos polares, opuestos. Así, cada concepto tiene un significado afectivo, o connotación, que varía a lo largo de tres dimensiones (evaluación, potencia y actividad, EPA). 
D+E: FUNDAMENTOS, PROBLEMÁTICA

Y SOLUCIONES PEDAGÓGICAS

Diferenciar la sensación y la impresión. Las acepciones del verbo sentir.

Otro concepto importante de diferenciar en este momento es el de sensación, cuya primera acepción en la RAE (2017) es la de impresión sensorial. Una impresión es un significado afectivo pasajero. Sin embargo hay impresión sensorial también. El verbo "sentir" posee múltiples acepciones que inducen a al estudiante a confundir lo que está sintiendo: ¿sí se trata de una emoción o no? Algunas de las acepciones más pertinentes (DRAE en línea) discutidas en el curso son:

1 tr. Experimentar sensaciones producidas por causas externas o internas. SENSORIAL

2 tr. Oír o percibir con el sentido del oído. Siento pasos. SENSORIAL

3 tr. Experimentar una impresión, placer o dolor corporal. Sentir fresco, sed. SENSORIAL

4 tr. Experimentar una impresión, placer o dolor espiritual. Sentir alegría, miedo. EMOCIÓN

5 tr. Lamentar, tener por doloroso y malo algo. Sentir la muerte de un amigo. EMOCIÓN, SENTIMIENTO

8 tr. Presentir, barruntar lo que ha de sobrevenir. U. especialmente hablando de los animales que presienten la mudanza del tiempo y la anuncian con algunas acciones. PREDICCIÓN

10 prnl. Padecer un dolor o principio de un daño en parte determinada del cuerpo. Sentirse DE la mano, DE la cabeza. SENSORIAL

11 prnl. Hallarse o estar de determinada manera. Sentirse enfermo. ESTADO DE ÁNIMO, ESTADO SOMÁTICO.

12 prnl. Considerarse, reconocerse. Sentirse muy obligado. JUICIO (DRAE, 2017)

Las 1, 2 y 3 se refieren a aspectos sensoriales; la 4 y 5 a aspectos concernientes a las emociones. La 11 es especialmente confusa ya que, frecuentemente, un estado de ánimo puede inducir cierta condición somática (p.ej. si tengo un estado de ánimo decaído me siento bajo de energía o inclusive, enfermo). La 12 habla de juicios sobre sí mismo que implican las propias actitudes, y aun los rasgos personales, que son fenómenos afectivos. 
D+E: FUNDAMENTOS, PROBLEMÁTICA

Y SOLUCIONES PEDAGÓGICAS

\section{Segunda sección: ¿Cómo se identifican los componentes de la emoción?}

En lo cotidiano existe una tendencia a identificar la emoción sólo con su sentimiento subjetivo y con expresiones del rostro, dejando por fuera otros componentes. Es por esto que se define la emoción como un fenómeno multicomponencial que involucra (Scherer, 2005):

- Un sentimiento subjetivo emocional

- Respuestas comportamentales (gestos faciales, movimientos, posiciones del cuerpo)

- Respuestas fisiológicas (frecuencia cardíaca, conductividad eléctrica de la piel, ondas cerebrales, secreción de hormonas, etc.)

- Una etiqueta o "label" de más duración: sentimiento

Esto es importante porque de esta forma se invita a los estudiantes a identificar dichos componentes. Además, se explica que si se desea medir una emoción, se pueden utilizar varios de sus componentes sin necesariamente descansar siempre en herramientas de auto reporte que poseen múltiples inconvenientes (Schwarz, 1999).

\section{Tercera Sección: ¿Cómo ordenar y estructurar las emociones?}

\section{Los circunflejos de emociones.}

Este es otro problema en un curso de D+E. La idea de que las emociones están ordenadas por opuestos de positivas-negativas se remonta a Aristóteles. Los circunflejos son un modelo dimensional de las emociones, ya que asumen que éstas pueden describirse según dos dimensiones, por ejemplo la valencia y la activación (Barrett \& Rusell, 2009). Aunque hoy en día existe una gran variedad de circunflejos en psicología (Russell, 1980), los circunflejos de emociones se han mostrado como uno de los constructos psicológicos más sólidos (Posner, Russell, \& Peterson, 2005). Estos han sido investigados en muchas culturas y lenguajes, y se han identificado aún en aquellas diferentes a las europeas (Plutchik, 2003). En general, los diversos circunflejos de emociones comprenden varios aspectos: Las emociones están ubicadas sobre la periferia de un círculo atravesado por dos ejes, considerando dos dimensiones (valencia positiva y negativa y nivel de activación o estimulación alto y bajo), con lo 
que una emoción particular, usualmente, tiene una emoción contraria, con signo opuesto en las dos dimensiones, ver Figura 3, aunque esta última afirmación es controversial ya que no necesariamente un circunflejo incluye toda la experiencia emocional. Si dos emociones están cerca en un circunflejo se entiende que son similares, (felicidad y alegría) mientras que si están separadas diametralmente (tristeza y felicidad, figura 3) se dice que su diferencia es máxima (Barrett \& Rusell, 2009).

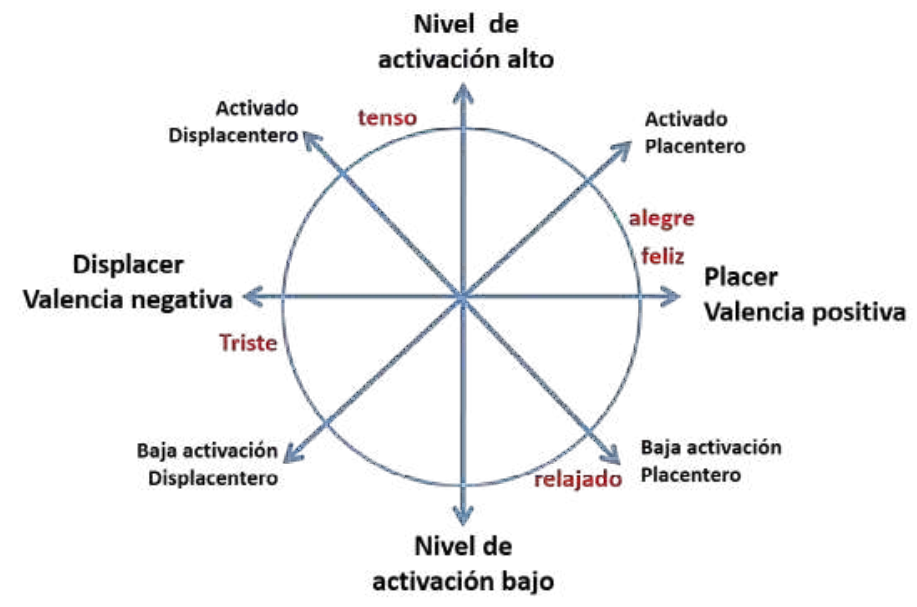

Figura 3. Un circunflejo de emociones, basado en Barrett \& Rusell (2009) y Plutchik (2003)

En un circunflejo existen unas emociones básicas, que son justamente aquellas representadas en los circunflejos simples, o en su círculo más interior, como sucede en circunflejos más sofisticados como el de Plutchick (2003). Las emociones no básicas, secundarias, pueden verse como una mezcla de dos emociones básicas adyacentes.

El modelo del circunflejo de las emociones tiene gran utilidad en un curso de D+E porque (Darbyshire, Bell, \& McDonald, 2006), aunque no explica cómo se forman las emociones, sí posee alto poder descriptivo ya que muestra cómo están ordenadas, señala que existen unas básicas y otras secundarias y llevan implícitas las dimensiones del núcleo afectivo (valencia positiva-negativa y nivel de estimulación alto y bajo). Con esto son de gran utilidad antes de mostrar el modelo de evaluación cognitiva de las emociones que explica eficazmente por qué, frente a un mismo producto, en dos personas pueden producirse diferentes emociones (Desmet 2008). 
D+E: FUNDAMENTOS, PROBLEMÁTICA

Y SOLUCIONES PEDAGÓGICAS

\section{Cuarta Sección: Los problemas disciplinares y epistemológicos}

Existen muchas teorías psicológicas sobre la emoción. Comprender y explicar qué son y cómo funcionan las emociones ha sido un problema abordado desde diferentes disciplinas, comprendiendo el individuo (psicología), el grupo (sociología) y su cultura (antropología: como varían las emociones culturalmente), y eso sin considerar los aspectos filosóficos. Strongman (2003) hace una presentación extensa de al menos 30 enfoques, modelos y teorías al respecto cubriendo dichos campos. Escoger entonces una teoría apta para abordar los problemas del D+E es en sí un desafío. Desmet (2002) hace un breve revisión en su tesis doctoral de al menos tres perspectivas: la evolutiva (concierne sobre todo la función de las emociones: para qué son), la fisiológica (relativa a la retroalimentación del cuerpo) concierne sobre todo la experiencia emocional; así, experimentar una emoción es resultado de un cambio corporal. Sin embargo, Desmet sostiene que este enfoque no dice cómo un estímulo externo genera una emoción. Finalmente Desmet revisa el enfoque cognitivo de las emociones planteado originalmente por Arnold en los años 60s. En este enfoque una emoción surge debido a un proceso de evaluación (o de appraisal', en inglés). Esta evaluación consiste en comparar un estímulo, en nuestro caso un producto, contra un interés personal (concern ${ }^{2}$ en inglés). Un interés es una preferencia por cierto estado de cosas en el mundo (Fridja, 2009), "Concern" en: (Sander \& Scherer, 2009). Hay muchos tipos de intereses entonces: un objetivo en una tarea (comprar harina para tortillas), una motivación (quiero adelgazar), un valor (me gusta comer sanamente), etc. Es entonces al evaluar un producto contra un interés particular que se presentará la emoción. Por ejemplo, un horno que consume poca energía, pero que es muy costoso, generará una emoción de expectativa o anticipación para alguien que valore mucho el vivir de forma ecológica, mientras que para alguien que posea intereses distintos, por ejemplo "que quepa en mi cocina" le generara apatía, desinterés o rechazo.

\footnotetext{
El término appraisal no posee una traducción exacta en español ya que un appraisal es a la vez una evaluación y un juicio. Aquí adoptaremos la traducción como "evaluación".

2 Concern tampoco posee una traducción precisa al español: puede ser un interés, una preferencia o algo que nos es importante. Lo asumiremos como "interés".
} 


\section{D+E: FUNDAMENTOS, PROBLEMÁTICA}

Y SOLUCIONES PEDAGÓGICAS

La perspectiva cognitiva de las emociones ha sido desde entonces ampliamente aplicada en diseño gracias su capacidad explicativa y predictiva. Un escollo importante que se ha debido sortear es el de la multitud de intereses que existen en nuestra relación con los productos, y por ende, de procesos de evaluación que pueden existir. Este problema lo empezó a estudiar Desmet (2009) proponiendo nueve fuentes en las que se originan las emociones, representadas en la siguiente matriz, Figura 4.

\begin{tabular}{|l|l|l|l|}
\hline $\begin{array}{l}\text { Etapa } \\
\text { de la interacción } \\
\text { con el producto }\end{array}$ & Actitudes & Objetivos & Valores y Normas \\
\hline & $\begin{array}{l}\text { Predisposiciones } \\
\text { hacia la categoría } \\
\text { del producto. } \\
\text { Creencias hacia } \\
\text { el producto }\end{array}$ & $\begin{array}{l}\text { Cosas que se desea ver listas } \\
\text { con la ayuda del producto. } \\
\text { Cosas que se desea ver pasar } \\
\text { con la ayuda del producto. }\end{array}$ & $\begin{array}{l}\text { Cómo creemos } \\
\text { individualmente y } \\
\text { socialmente que deben ser } \\
\text { las cosas. }\end{array}$ \\
\hline $\begin{array}{l}\text { Apariencia } \\
\text { del producto }\end{array}$ & & & \\
\hline Uso del producto & & & \\
\hline $\begin{array}{l}\text { Consecuencias del } \\
\text { uso del producto }\end{array}$ & & & \\
\hline
\end{tabular}

Figura 4. Matriz de las nueve fuentes de emociones con los productos (Desmet et al., 2007).

El problema de la multiplicidad de intereses, y en consecuencia, de evaluaciones, ha sido atacado posteriormente por Desmet y sus colegas de manera a proponer patrones de evaluación recurrentes en una gran diversidad de situaciones y eventos en los que intervienen humanos interactuando con diferentes categorías de productos (Demir, Desmet, \& Hekkert, 2009). Este trabajo fundamenta una de las herramientas del curso, como se presentará más abajo.

De interés disciplinar es el ofrecer una definición del D+E, (Figura 5). El diseño para las emociones es una parte del diseño basado en el usuario que busca generar en éste emociones adecuadas en la interacción con el producto, que respondan a: 
- Lo esperado y deseado por el usuario

- Lo buscado por el diseñador

- Lo que busca la estrategia de producto y marca.

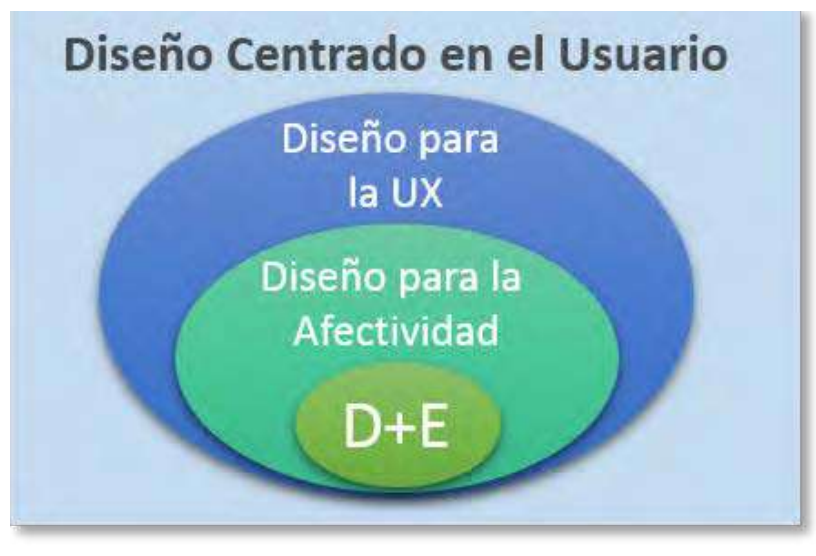

Habiendo hecho un recorrido por los diferentes problemas vinculados a la enseñanza aprendizaje del D+E pasamos entonces a exponer las virtudes y problemas encontrados en la implementación de herramientas utilizadas en el curso de D+E. En diseño frecuentemente se proponen nuevos métodos y herramientas, pero son escasamente aplicadas en la práctica. En este artículo, por el contrario, se muestran experiencias en donde se conciben y/o aplican repetidamente dichos métodos, mostrando así a los estudiantes el cómo integrar la teoría en sus procesos de diseño.

\section{Los Métodos y Herramientas en un curso de D+E}

A continuación se exponen una variedad de herramientas, desde altamente heurísticas hasta altamente estructuradas, en el mismo orden en que se enseñan en el curso. Se mostrarán diversos ejemplos resultantes de su aplicación. 
D+E: FUNDAMENTOS, PROBLEMÁTICA

Y SOLUCIONES PEDAGÓGICAS

Existen varios problemas que enfrenta un diseñador o jefe de proyecto al escoger un método de diseño para la UX y el D+E (Maya, 2010). Primero, la dificultad de definir una experiencia particular con el producto o uno de sus niveles; segundo, la continua aparición de nuevos métodos y enfoques, y tercero, las incoherencias conceptuales y de lenguaje entre los métodos, debido a su origen disciplinar diferente. Al escoger las herramientas a utilizar en el curso se hizo énfasis en que identificaran de manera clara y bien diferenciada la experiencia emocional, no sólo frente a otros niveles de la experiencia, sino también respecto a otros fenómenos afectivos. Además, se presentaron herramientas basadas en tradiciones de investigación bien consolidadas y que contasen con resultados robustos, tal como sucede con los trabajos del ID studio Lab de TUDelft. Se buscó mostrar una diversidad de enfoques, que por otro lado, diese cuenta de un campo que se encuentra aún con una multitud de enfoques y métodos en competición, i.e., que se encuentra en un estado pre-paradigmático. Finalmente, el último criterio de selección responde al tríptico costo-calidad-plazo de entrega, esencial en el desarrollo de nuevos productos: los métodos a enseñar deben responder a un abanico de requerimientos. Primero, precisión y/o robustez (por ejemplo, métodos heurísticos, poco precisos vs KE, muy precisa). Segundo, en términos de recursos de conocimiento especializado (los métodos heurísticos requieren poco conocimiento especializado, la KE, mucho (Nagamachi \& Lokman, 2010). Tercero, los costos, e.g., los métodos heurísticos no exigen ninguna investigación de terreno, usualmente costosa, mientras que la KE exige estudios de usuarios con extensas muestras de población (200 personas o más). Cuarto y último, la facilidad de aplicación va asociada al plazo de entrega del proyecto ya que algunos métodos dan resultados casi inmediatamente, mientras que otros, como el método de diseño para el Wow de Desmet, pueden exigir semanas o en el caso de la KE, meses.

El proyecto de investigación europeo Engage Consortium (2006), integrando diseñadores, investigadores e industria, desarrolló una base de datos consultable en Internet sobre métodos para el $\mathrm{D}+\mathrm{E}$, y más ampliamente para la UX. La base utiliza una herramienta desarrollada en el proyecto, que apoya la selección de los métodos. La base de datos contiene unos 60 métodos que son seleccionados a través de un gran número de filtros. Es posible acceder a través de una membresía de la Design and Emotion society. 
Para enfrentar el problema de qué métodos deben enseñarse en el curso, se ofrece entonces una combinación de métodos de base heurística y métodos semiestructurados y estructurados. Los conceptos básicos presentados sirven para que los alumnos hagan un manejo eficiente de ellos. Antes de iniciar la aplicación de las herramientas se debe definir un tipo de producto específico, que será la base del rediseño, así como un usuario. Es deseable que el producto provenga de la cotidianeidad de los estudiantes y que sea técnicamente simple. Por ejemplo, el estuche para Smartphone de la Figura 6. Todos los métodos se han presentado en español.

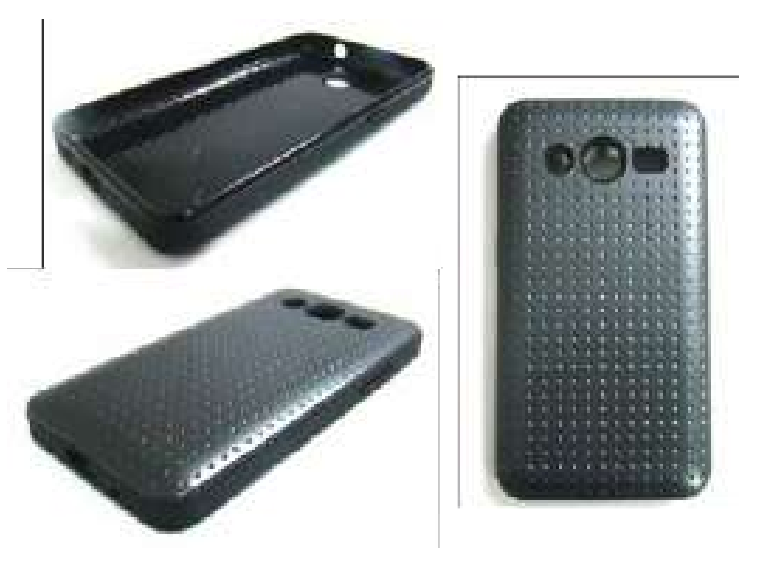

Figura 6. Estuche plástico protector para Smartphone utilizado como base para el rediseño usando herramientas de D+E. Foto: Ever Patiño y Simón Ríos.

\section{Los heurísticos para la generación de apego}

Los heurísticos de apego han sido desarrollados basándonos en el trabajo de (Mugge, Schoormans, \& Schifferstein, 2008). El apego como fuente de placer ha sido investigado por (Klcinc \& Baker, 2004; Mugge, Schifferstein, \& Schoormans, 2010; Schifferstein \& Zwartkruis-Pelgrim, 2008). Sentimos apego cuando un producto continúa generándonos emociones positivas por largo tiempo después de haber sido adquirido. El apego es interesante como estrategia ya que si el producto llega a su fin de vida o el usuario lo pierde, es bien probable que recompre la marca o el mismo producto original (Mugge et al., 2010; Schifferstein \& Zwartkruis-Pelgrim, 2008). La figura 7 muestra bocetos originales de aplicación de los heurísticos de apego. Dichos heurísticos pueden clasificarse en cuatro tipos. 
D+E: FUNDAMENTOS, PROBLEMÁTICA

Y SOLUCIONES PEDAGÓGICAS

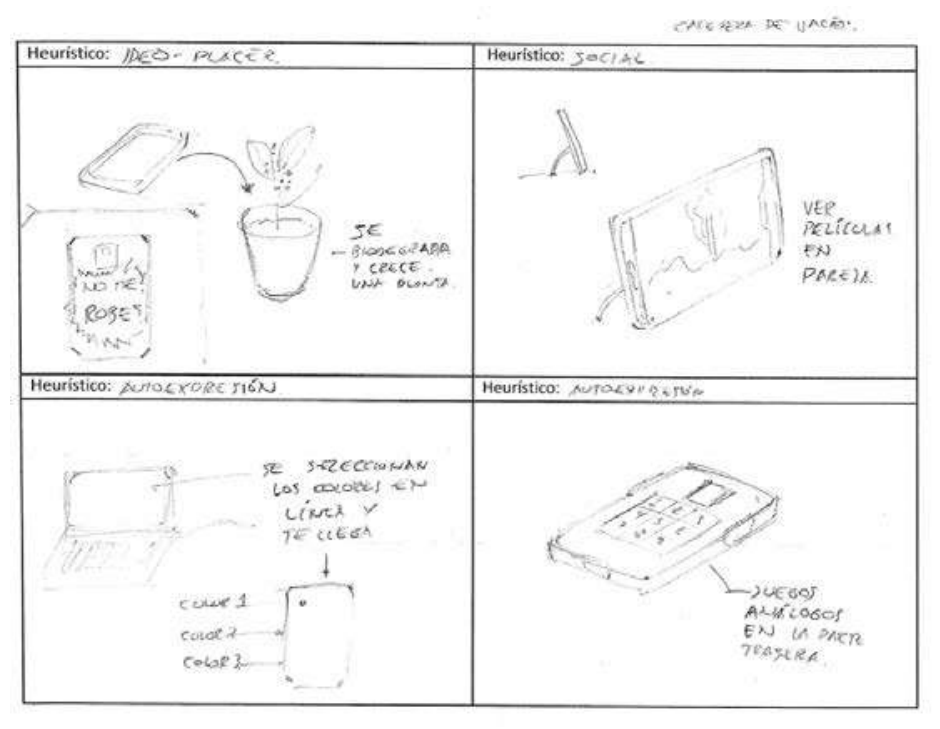

Figura 7. Bocetos resultantes de la aplicación de heurísticos de apego en el caso del estuche para celular. Figura y ejemplo por Ever Patiño y Simón Ríos.

Apego a través de la generación de placer. Estos se basan en los cuatro tipos de placer expuestos por Tiger (1992). A partir de la definición de cada uno de estos placeres se les invita a generar ideas para que el producto que se está diseñado produzca dicho placer en el usuario.

- Placer sensorial | haga su producto agradable estéticamente.

- Placer psicológico | haga que el producto realice mejor sus funciones. Haga que el producto tenga funciones que otros productos similares no tienen (de forma más o menos exclusiva), y/o haga que el producto tenga un elemento que genere sorpresa agradable; Figura 8.

- Haga que el producto genere ideo-placer por estar de acuerdo con valores que le son importantes al usuario.

- Haga que el producto genere socio-placer por incitar a la conversación alrededor de él. 
D+E: FUNDAMENTOS, PROBLEMÁTICA

Y SOLUCIONES PEDAGÓGICAS
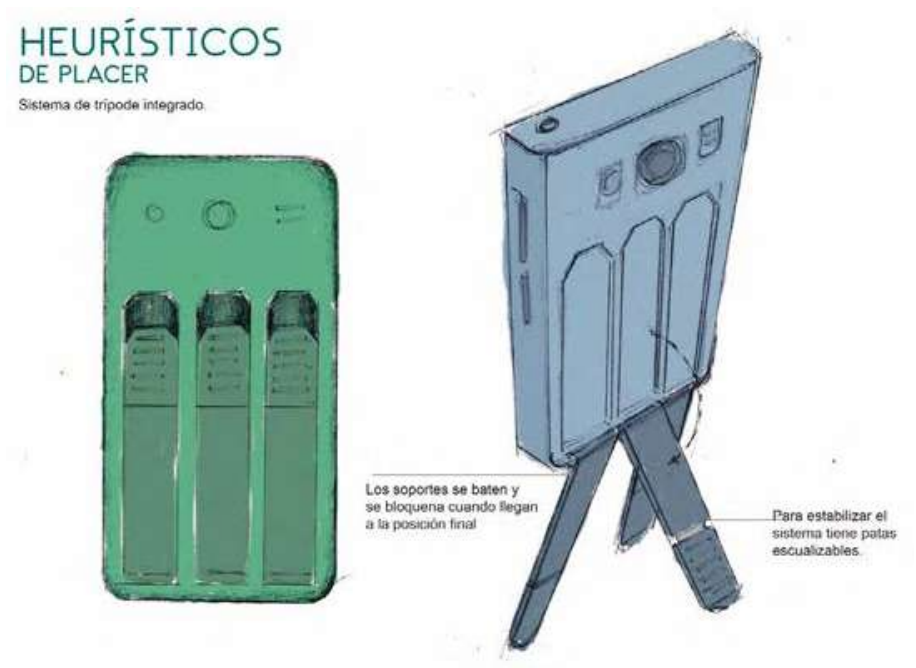

Figura 8. El estuche posee un trípode integrado con el cual posicionar el Smartphone de manera visible y para que se pueda escuchar música. Ejemplo y figura por Ever Patiño y Simón Ríos, 2017.

Apego a través de productos que facilitan el que el usuario se diferencie de otras personas y que exprese su identidad. Estos son los Heurísticos de Autoexpresión.

- Heurísticos de personalidad del producto | Traduzca características de la personalidad del usuario en características del producto.

- Heurístico de personalización | Haga que el producto se deje personalizar, haciendo que el usuario lo haga más único y personal (e.g., la motocicleta Harley Davidson que es personalizada por cada dueño).

- Haga parte al usuario en el proceso de diseño | Por ejemplo, El Renault Kangoo, fue diseñado preguntándole a los niños cómo sería su automóvil ideal: como si fuese una pecera con grandes ventanas para poder ver todos los alrededores.

- El heurístico de customización del producto / haga que el producto se pueda customizar desde la fábrica. Por ejemplo, hoy en día un automóvil ofrece decenas de posibilidades distintas de equipamiento desde la fábrica. 


\section{Heurísticos que permiten la filiación grupal.}

- Hacer que el usuario se sienta unido, conectado e involucrado con un grupo de personas a través de productos que serán compartidos con otros en una actividad grupal. Como ejemplo tenemos las motocicletas Harley-Davidson, las motonetas Vespa, o los automóviles Mini. Los usuarios de estos vehículos con frecuencia hacen parte de clubes y comunidades que comparten actividades e intereses alrededor de su coches. Ver figuras 9 y 10 .

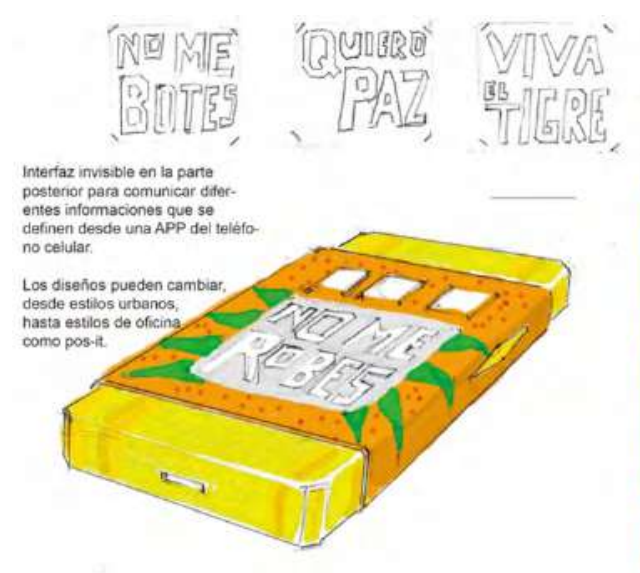

HEURÍSTICOS DE AUTO-EXPRESIÓN

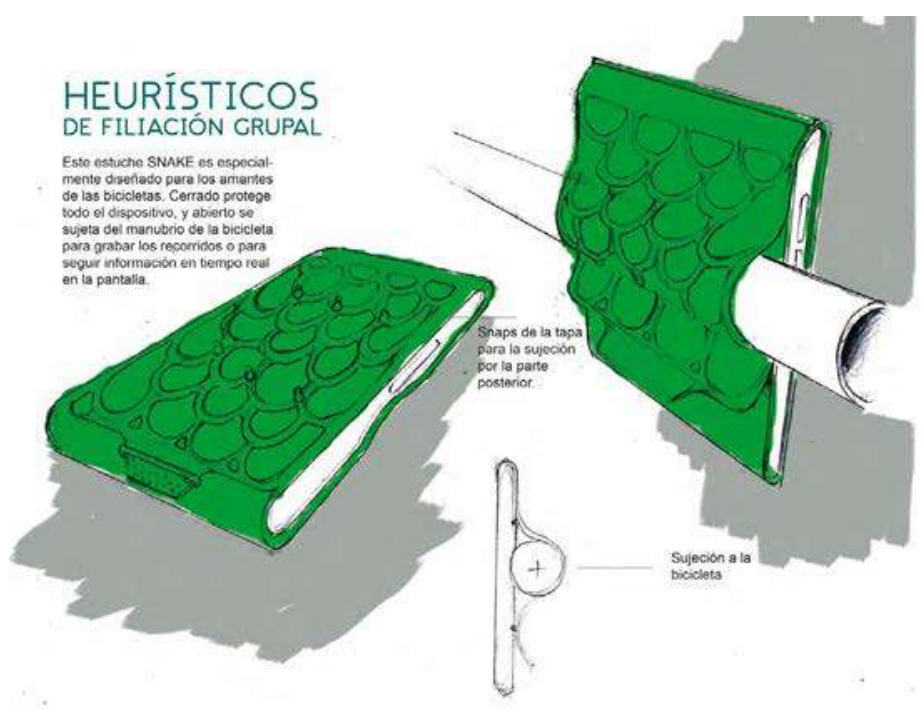

Figura 9. Heuristicos de autoexpresión. Se presenta una pantalla o interfaz en el estuche, que se activa cuando lo desee el usuario y que comunica diferentes informaciones que éste desee. Estas informaciones se pueden definir desde una app en el Smartphone. Ejemplo y figura por Ever Patiño y Simón Ríos, 2017.
Figura 10. Este estuche "Snake" está especialmente diseñado para los amantes de las bicicletas. Cuando está cerrado protege todo el dispositivo y abierto se sujeta del manubrio para grabar recorridos con la cámara del celular o para seguir informaciones del GPS o de internet en tiempo real. Ejemplo y figura por Ever Patiño y Simón Ríos, 2017. 
D+E: FUNDAMENTOS, PROBLEMÁTICA

Y SOLUCIONES PEDAGÓGICAS

Heurísticos para generar apego permitiendo los buenos recuerdos.

- Introducir olores evocadores en los productos. La caja de galletas Mary Biscuit de Alessi que huele a vainilla es una ilustración de este heurístico, figura 11.

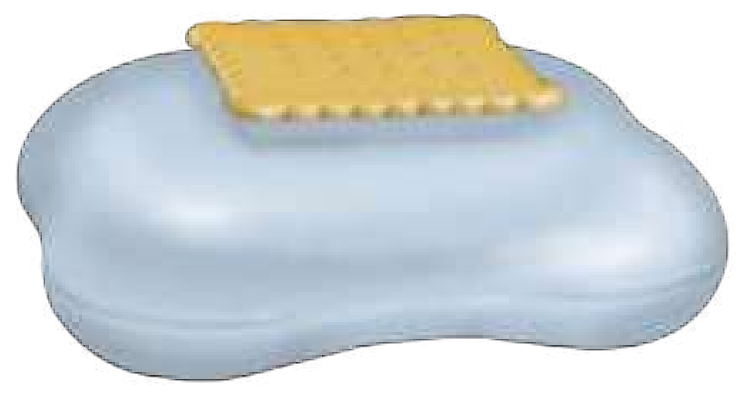

Figura 11. La caja de galletas Mary Biscuit de Alessi posee un olor integrado al material plástico que evoca las galletas de vainilla de antaño. Ilustración: David Schoenwald, Licencia Creative-Commons 3.0

Permitir que el producto envejezca dignamente gracias a sus materiales (Chapman, 2009). Utilizar entonces materiales resistentes al desgaste superficial o texturas, en los plásticos, que oculten el rayado. En un trabajo en desarrollo, por ejemplo, Bridgens et al. (2015) investigaron cómo estuches de celular en materiales tales como cuero, corcho, plástico, caucho, titanio, castaño y bambú eran percibidos estética y semánticamente al envejecer y verse deteriorados. Esto puede dar indicaciones para apoyar la aplicación del heurístico.

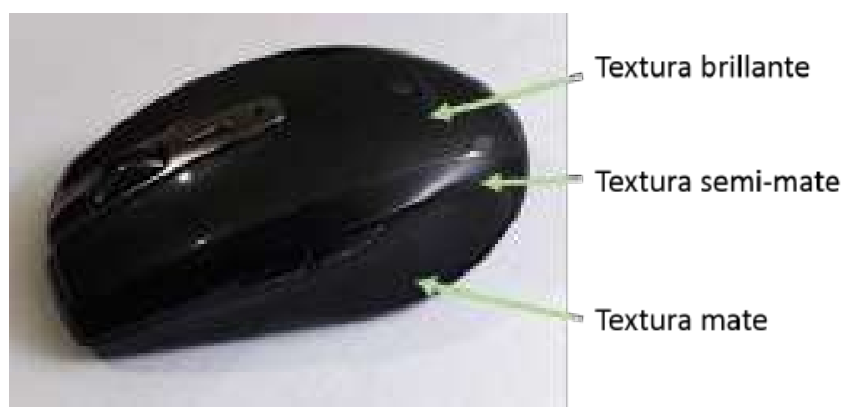

Figura 12. El uso de plásticos con textura mate o semi mate evita que el producto se deteriore ostensiblemente en zonas de alto contacto con la mano del usuario. Mouse Logitech. [Fotografía por el autor]. 
Herramienta de los patrones de evaluaciones cognitivas.

Esta herramienta se basa en el trabajo de Demir, Desmet et al. (2011). Para enfrentar la complejidad que plantea el gran número de intereses (concerns) y evaluaciones cognitivas (appraisals), ellos identificaron los patrones de evaluación que permiten generar ciertas emociones con los productos.

Las emociones más frecuentes identificadas en la interacción con los productos fueron:

\begin{tabular}{|c|c|}
\hline Felicidad & Alegría \\
\hline Satisfacción & Contento \\
\hline Rabia & Irritación \\
\hline Desengaño & Insatisfacción \\
\hline
\end{tabular}

Demir et al. (2011) presentan tablas de intereses para las cuatro parejas de emociones. Por ejemplo, la tabla aquí debajo, figura 13, presenta los tipos de intereses y ejemplos para felicidad y alegría.

\begin{tabular}{|l|l|l|}
\hline $\begin{array}{l}\text { Nivel } \\
\text { del } \text { interés }\end{array}$ & Tipo de Interés (concern) & Ejemplos del interés (concern) \\
\hline $\begin{array}{l}\text { Motivo } \\
\text { general }\end{array}$ & Pertenencia social & Chatear con mi familia a través de la webcam \\
\hline \multirow{5}{*}{} & Interacción social & Recibir un SMS chévere \\
\cline { 2 - 3 } & Desafío físico & Montar en patineta \\
\cline { 2 - 3 } & Cuidarse uno mismo & Cepillarse los dientes con un cepillo electrónico \\
\cline { 2 - 3 } & Estimulación intelectual & Analizar el mecanismo "mágico" de un precolador de café \\
\cline { 2 - 3 } & Ser independiente y poderoso & Tener un Jeep \\
\cline { 2 - 3 } & Sentirse en familia y seguro & Ser capaz de abrir la cerradura de un apartamento nuevo \\
\cline { 2 - 3 } & Ser bonito & Las consecuencias de ponerse pestañas postizas \\
\hline
\end{tabular}

Figura 13. Tablas de intereses y sus ejemplos [Elaboración propia]. 
D+E: FUNDAMENTOS, PROBLEMÁTICA

Y SOLUCIONES PEDAGÓGICAS

Con base en estas tablas se generó la herramienta y sus formatos de apoyo, como se ve aquí debajo, figura 14.

\begin{tabular}{|l|c|l|}
\hline Tipo de interés (concern) & $\begin{array}{l}\text { Emociones que } \\
\text { contribuye a generar }\end{array}$ & Cómo hacerlo en mi producto \\
\hline $\begin{array}{l}\text { Pertenencia social: mi producto debe } \\
\text { ayudar a identificarme o pertenecer a algún } \\
\text { grupo social (mi clase, mi grupo de amigos, } \\
\text { mi universidad,...) }\end{array}$ & Felicidad y alegría & \\
\hline $\begin{array}{l}\text { Interacción social: mi producto debe } \\
\text { ayudarme a facilitar la interacción social }\end{array}$ & Felicidad y alegría & \\
\hline $\begin{array}{l}\text { Desafío físico: mi producto debe ayudarme } \\
\text { a lograr algún desafío físico que me surja } \\
\text { o me imponga }\end{array}$ & Felicidad y alegría & \\
\hline $\begin{array}{l}\text { Cuidarse uno mismo: mi producto debe } \\
\text { contribuir a mi apariencia personal }\end{array}$ & Felicidad y alegría & \\
\hline
\end{tabular}

Figura 14. Formato de la herramienta de D+E para integrar los intereses del usuario [Elaboración propia].

Esta herramienta es interesante porque obliga al estudiante a pensar en cómo el tipo de producto que está (re)diseñando se puede mejorar, desde el punto de vista del usuario, para que genere un conjunto bien definido de emociones. En el caso se muestra la tabla para las emociones: "Felicidad" y "Alegría". El estudiante relaciona un interés específico, por ejemplo, "Pertenencia social: mi producto debe ayudarme a identificarme o pertenecer a algún grupo social (mi clase, mi grupo de amigos, mi universidad,(...)" (ver figura 15), y si dicho interés es pertinente para el producto, puede generarse la emoción felicidad y alegría en el usuario. La mayor dificultad de este método estriba en hacer pensar al estudiante en el cómo: es allí donde debe entrar a actuar su creatividad. El estudiante debe pensar en elementos físicos del producto, ya sean funcionales, de usabilidad, semánticos y/o estéticos, que faciliten que el interés se cumpla. Otra dificultad está en que a veces la categoría de producto definitivamente no tiene que ver con el interés del caso, con lo que la aplicación del interés es muy difícil o imposible. 
D+E: FUNDAMENTOS, PROBLEMÁTICA

Y SOLUCIONES PEDAGÓGICAS
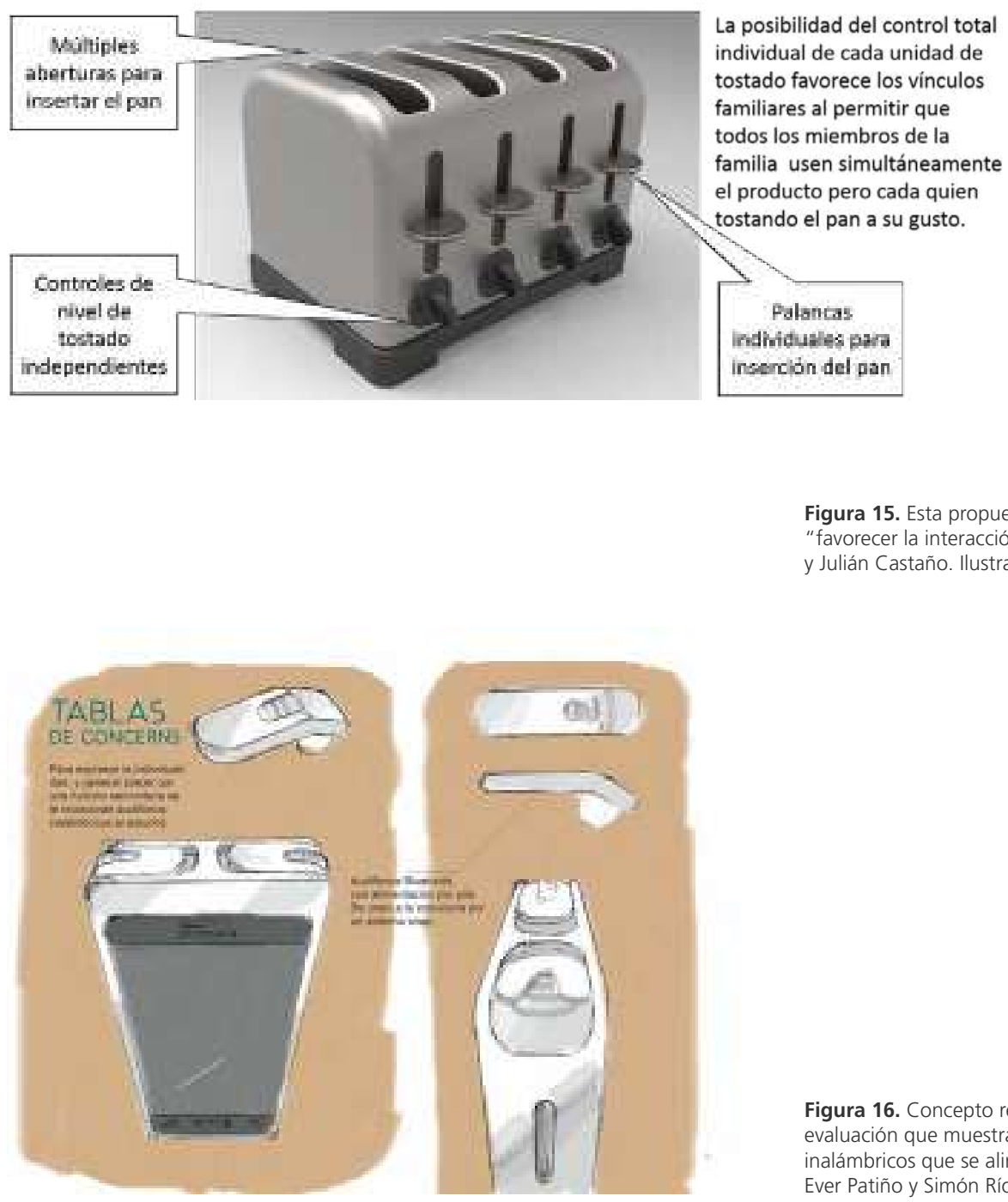

Figura 15. Esta propuesta de tostadora fue creada dando respuesta al interés "favorecer la interacción con mi familia". Ejemplo: Daniel Paris, Felipe Álvarez y Julián Castaño. Ilustración: María Isabel Vargas P.

Figura 16. Concepto resultante de la herramienta de los patrones de evaluación que muestra el estuche del celular integrando audífonos inalámbricos que se alimentan de la batería del celular. Ejemplo e ilustración: Ever Patiño y Simón Ríos.

\section{Herramienta el continuo de la experiencia.}

Uno de los puntos clave en un curso de D+E es el hacer caer en cuenta al estudiante de que las emociones que sentimos con los productos están asociadas a los diferentes momentos de la interacción. Primero que todo está el reconocimiento de las etapas clásicas de la interacción: antes (anticipación), durante, después y, posiblemente, mucho después. Esta última etapa es difícil de abordar en un curso de corta duración. Sin embargo, con esta herramienta lo que se logra con relativa facilidad es asociar a cada momento de la in- 
D+E: FUNDAMENTOS, PROBLEMÁTICA

Y SOLUCIONES PEDAGÓGICAS

teracción una emoción, si ésta se presenta, y hacer caer en cuenta al estudiante que dichas emociones están vinculadas a la usabilidad y funcionalidad del producto.

Esta herramienta consiste entonces en un diagrama que representa un continuo temporal de la interacción con el producto y la valencia positiva y negativa de las emociones, ver figura 17. Sobre el diagrama se van representando las emociones asociadas a cada uno de los pasos de la interacción con el producto, que son reportadas por el producto. Esto se acompaña de fotografías de la interacción. Normalmente, las emociones positivas o negativas intensas se generan por aciertos o fallas importantes en la funcionalidad o usabilidad del producto; de otra forma, se trata de emociones de baja intensidad (Desmet 2004).

El usuario investigado debe ser entrenado previamente en el protocolo de pensar en voz alta (Think aloud protocol). Esto se hace de la manera indicada en la literatura, resolviendo por ejemplo en voz alta un acertijo del tipo de los caníbales y/o cabras que deben pasarse de una orilla a otra de un río.

De esta forma el usuario expresará sus pensamientos sobre la interacción develando los problemas e inconvenientes que encuentre. A la vez, irá reportando sus emociones cuando el investigador se lo demande. Consecuentemente, el usuario debe ser familiarizado de antemano con el instrumento de reporte y medición de emociones que se vaya a usar. Para el efecto se han utilizado con éxito los 12 rostros de los personajes de PrEmo, Product Emotion Measure, (herramienta de medición de emociones desarrollada por Desmet, 2005), cada uno de ellos expresando una emoción diferente. Otro instrumento, de muy sencilla aplicación, es el circunflejo de Plutchick, ya que su forma gráfica multicolor es clara para el encuestado; la rueda de emociones de Ginebra (Geneve Emotion Wheel, Scherer, 2005) también se ha aplicado. 
D+E: FUNDAMENTOS, PROBLEMÁTICA

Y SOLUCIONES PEDAGÓGICAS

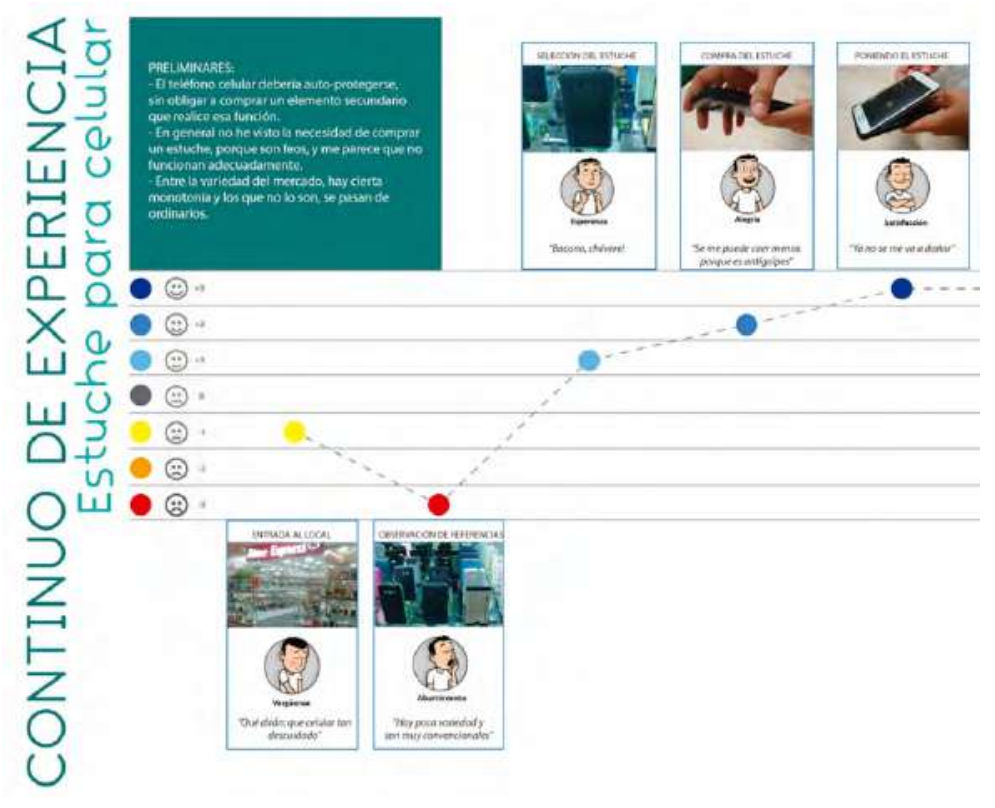

Figura 17. Fragmento del continuo de la experiencia en el caso del estuche para Smartphone: las caricaturas corresponden a las emociones de PreMo. Ejemplo e ilustración: Ever Patiño, Simón Ríos.

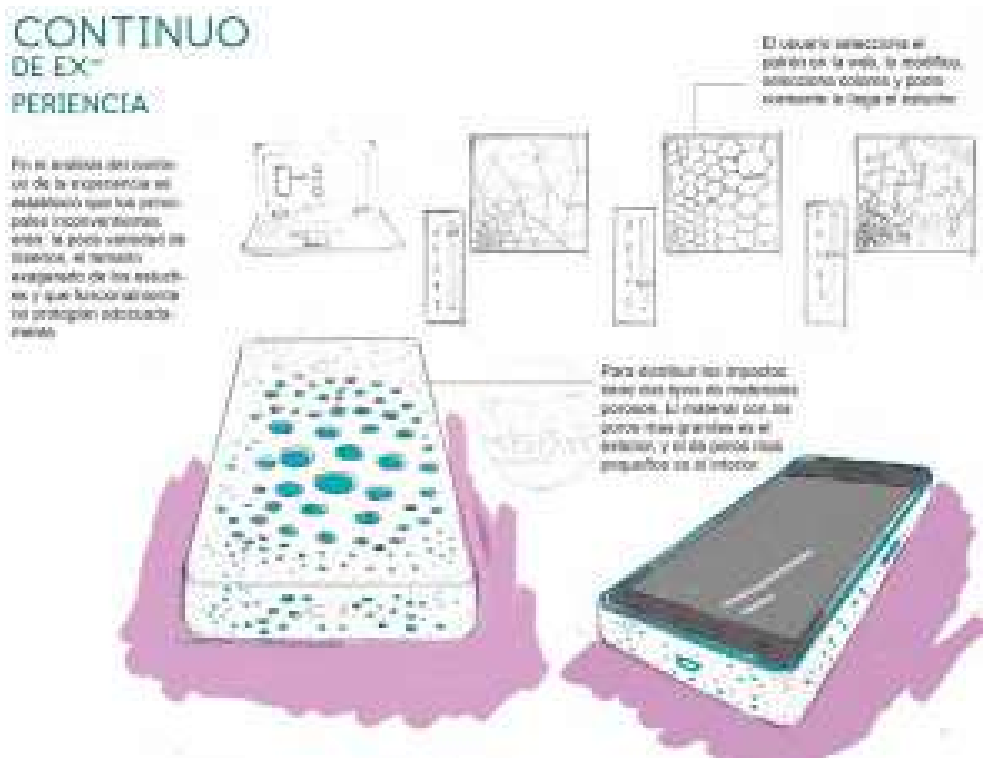

Figura 18. Conceptos para el estuche obtenidos de la herramienta del continuo de la experiencia, basados en la poca variedad de formas del estuche y que funcionalmente no protegían adecuadamente el celular. El uso de dos materiales porosos facilita la absorción de impactos. El usuario puede seleccionar en la web el patrón deseado, modificándolo en su geometría y colores. Ejemplo e ilustración: Ever Patiño, Simón Ríos. 


\section{El Método TADA}

Este método, desarrollado por Grimaldi (2008), busca diseñar productos que generen sorpresa positiva en el usuario. La sorpresa positiva al acompañarse de una alta activación, hace que la persona esté muy atenta a los estímulos y que las experiencias generen una memoria fuerte en la persona. El método consiste en los siguientes pasos:

- Identificar las principales características del producto.

- Identificar cuáles de éstas son esenciales: se trata de una característica que tiene que tener el producto para ser identificado como de su clase. Por ejemplo, una lámpara que no ilumina, independientemente de su tecnología o forma, dejaría de ser una lámpara.

- Cambiar una de las características no esenciales de forma radical, y violando expectativas que tenga la persona sobre el producto: esto originará una sorpresa negativa. Entre más familiar sea el tipo de producto para el usuario, más fuertes serán sus expectativas. Por ejemplo, las lámparas son frágiles, de vidrio, o metal, poseen una fuente de energía, etc.

- El conflicto que surge al alterar la característica no esencial debe resolverse de manera positiva, o que genere sorpresa positiva.

Es importante situar el todo en un escenario en donde un observador vea el producto de lejos y se sienta intrigado. Así, al acercarse, la persona notará la incongruencia. Se produce la sorpresa negativa entonces cuando la interacción es cercana. Dicha sorpresa se torna en sorpresa positiva al resolverse la incongruencia. 


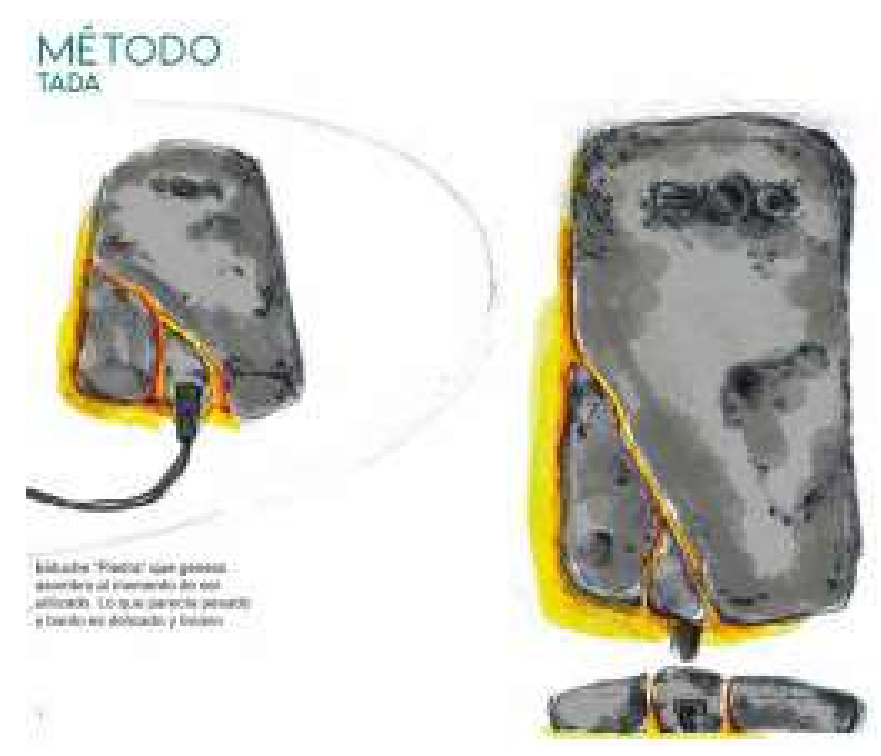

Figura 19. Aplicación del método Ta-Da. Boceto del estuche de celular "Piedra" parece burdo y pesado al ser visto desde lejos. Sin embargo, al ser utilizado se revela como suave y liviano. Ejemplo e ilustración por Ever Patiño y Simón Ríos.

Este método es un poco desafiante para los estudiantes. Su mayor dificultad está en la última parte, al deber imaginar y/o identificar el escenario y situación en la que se presenten y resuelvan las incongruencias. Ver Grimaldi (2008) para otros ejemplos de su aplicación.

Una alternativa a la generación de sorpresa positiva en el usuario se presentó en el curso a través de la generación de incongruencia sensorial basándose en la apariencia visual de los materiales, según los mecanismos definidos por Ludden (2008). Este enfoque, aunque muy promisorio, fue abandonado después de dos oportunidades debido a la dificultad para identificar los materiales (usualmente materiales avanzados desde el punto de vista sensorial) que permitan generar la incongruencia sensorial. Como ayuda, se utilizó la base de datos de materiales Material Connexion, (materialconnexion.com/) que produce fichas mensuales de los nuevos materiales en el mercado. Sin embargo, al no tener acceso sensorial directo a dichos materiales, la mera generación de ideas de producto se hacía muy difícil. 


\section{El método semiestructurado de Desmet et al., de diseño para el Wow.}

Este método se expone en el curso a manera de ejemplo de un método que combina herramientas estructuradas con un proceso creativo menos estructurado. Al requerir técnicas estadísticas como el ACP y el software PrEmo su aplicación puede ser relativamente compleja. A parte de esto, el método presenta mucho potencial de aplicación, inclusive para diseñar para otras emociones o combinaciones de emociones.

\section{La Ingeniería Kansei: método altamente estructurado}

La KE se presenta en el curso como un ejemplo de un enfoque altamente estructurado para el D+E. La KE es muy vasta, siendo una disciplina por sí misma, con lo que en el curso sólo es presentado el método general de la KE, enfatizando el concepto de la expansión del espacio de propiedades del producto y la expansión del espacio semántico. También se presenta un ejemplo de aplicación manual de la KE (la llamada KE Tipo 1), sin entrar en detalles sobre otros tipos de KE, mucho más complejos y sofisticados (tipos 2 a 5).

\section{La propuesta definitiva:}

A continuación, en un ejemplo desarrollado por los estudiantes del curso, Ever Patiño y Simón Ríos, se presenta el concepto final del estuche para celular que integra elementos de los conceptos de producto anteriormente presentados. Vulcano es un estuche protector en silicona para el smartphone Samsung DUO, figura 20.

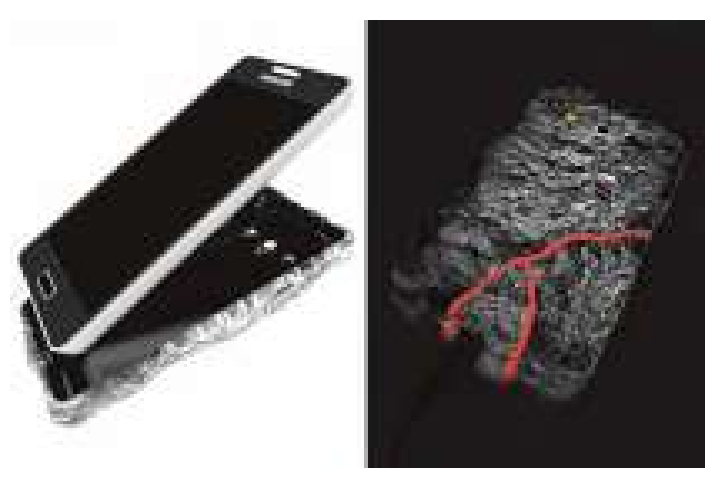

Figura 20. Vulcano, el concepto final del estuche para Smartphone, toma varias de las ideas desarrolladas con el método TADA. Está fabricado en silicona inyectada y su aspecto de roca se logra con water transfer (hidrografía), logrando una apariencia de alto detalle. De lejos se percibe como una roca, burda y pesada; sin embargo, un elemento luminosos aparece como incongruente.

Ejemplo e ilustración: Ever Patiño, Simón Ríos. 
D+E: FUNDAMENTOS, PROBLEMÁTICA

Y SOLUCIONES PEDAGÓGICAS
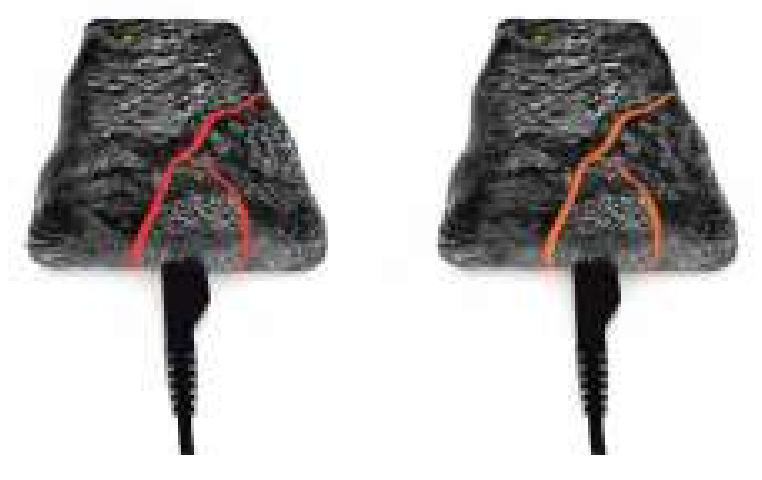

Figura 21. Al recargar el celular, la fisura se activa y genera una luz desde el amarillo hasta el rojo, de manera continua, cíclica y lenta. Esto añade un aspecto lúdico a un momento que normalmente es ausente de emociones positivas. Ejemplo e ilustración: Ever Patiño, Simón Ríos.

\section{Discusión}

Se discutirá el desarrollo del curso en los términos generales planteados por el esquema ADDIE.

El análisis ha permitido establecer los objetivos del curso en función del público. Así, el general es: "diseñar productos y servicios innovadores a través de la aplicación de métodos del D+E y de una comprensión de cómo funcionan las emociones de los usuarios". En la fase de diseño se definieron los temas teóricos y las herramientas y /o métodos de diseño a utilizar, teniendo en cuenta las capacidades de los alumnos y el bajo tiempo de clase disponible (16 horas en total). En el desarrollo se planteó el programa del curso haciendo uso de ciertas herramientas que fueron descartadas en el tiempo y cambiadas por otras, fruto de resultados de investigación más definitivos y robustos. Este fue el caso de la eliminación de la herramienta Matriz de las nueve fuentes de emociones, que se reemplazó por la herramienta de los patrones de evaluación. Así entonces, durante la Implementación, numerosas modificaciones se han hecho sucesivamente. En la Evaluación, en la práctica es a veces difícil llevar un curso de esta naturaleza hasta el punto de que presente una estabilidad en sus contenidos. En un dominio que está en constantes cambios, como lo es el $\mathrm{D}+\mathrm{E}$, puede tomar mucho tiempo. Esto es un requisito para hacer una evaluación estructurada, tanto cualitativa como cuantitativa que tenga en cuenta los 
D+E: FUNDAMENTOS, PROBLEMÁTICA

Y SOLUCIONES PEDAGÓGICAS

resultados del curso en un plazo de tiempo razonable. Un proyecto de dicha naturaleza puede asumirse como una investigación interesante para quienes trabajen la pedagogía del diseño industrial y del $\mathrm{D}+\mathrm{E}$.

De otro lado, aunque la opinión de los estudiantes sobre el curso no ha sido recogida sistemáticamente, los comentarios y la actitud general hacia el curso son muy positivos. Los elementos teóricos presentados les muestran que aunque las emociones son subjetivas, no es imposible diseñar para ellas; esto gracias a que el mecanismo que las produce es universal. Los alumnos traen al curso la creencia popular de que no se puede diseñar de forma estructurada aquello que es subjetivo; sin embargo, su propio trabajo contribuye a desmontar dicha creencia. El énfasis práctico-aplicado del curso también contribuye a su buena recepción. Una pregunta generalizada es por qué el curso no se da como materia obligatoria en los currículos de pregrado. La respuesta que se suele dar es que los currículos ya están estructurados con cursos desde una visión más clásica del diseño y la ingeniería, con lo que un campo nuevo, como es el $\mathrm{D}+\mathrm{E}$, todavía no se considera básico.

La dificultad de implementación de las herramientas ha sido variada. La herramienta de la matriz de nueve fuentes de emociones con los productos (Figura 4) se retiró debido a que su comprensión y aplicación era muy difícil para los alumnos, aunque algunas veces dio "insights" interesantes. Por el contrario la herramienta de los patrones de evaluación, una vez entendido su mecanismo, funciona sin problemas. El método TaDa ha sido especialmente difícil debido a que es un reto para los alumnos imaginar una historia y un contexto en el que la sorpresa negativa debe transformarse en positiva. TaDa exige ser muy analítico al principio, al identificar la propiedad esencial del objeto, para poder cambiar otra que no sea esencial. Luego exige bastante imaginación para desarrollar la narración. Es un método que involucra muchos elementos diferentes y que son novedosos para los alumnos, con lo que su aplicación a veces presenta dificultades. Otra herramienta que presenta algunas dificultades es el continuo para la experiencia ya que es necesario entrenar algunos de los alumnos en la técnica de pensar en voz alta. Se sabe que es una habilidad en la que solo algunas personas son aptas (Ericsson; Simon, 1993) con lo que a veces una capacidad 
limitada para narrar el flujo del pensamiento interfiere con la herramienta. Nunca se han identificado interferencias debidas a que las herramientas hayan sido concebidas en culturas europeas.

La herramienta del continuo de la experiencia brinda un acercamiento a la medición de las emociones ya que el sujeto estudiado reporta sus emociones y un nivel de intensidad correspondiente, tal como se hace en la herramienta PrEmo. Medir las emociones es importante para un D+E estructurado, ya que es el mecanismo de control para constatar que el producto diseñado sí cumpla con los requerimientos afectivos -emocionales del usuario. La medición de emociones es entonces un capítulo importante del D+E. Actualmente muchas herramientas se han propuesto midiendo diferentes indicadores correspondientes a componentes comportamentales (p.ej. expresiones del rostro) y/o fisiológicos (por ejemplo, conductividad eléctrica de la piel, EEG); sin embargo, las herramientas de auto reporte siguen siendo las más utilizadas debido a su facilidad de aplicación y de interpretación y análisis de resultados. En un curso corto como el aquí descrito sólo se han ofrecido diferentes ejemplos con PrEmo y con el circunflejo de Plutchick. Más ampliamente, también se ha usado el diferencial semántico para medir los tres niveles afectivos de la UX. Sin embargo, la preparación de las escalas, aunque es un ejercicio relativamente simple, es bastante dispendioso cuando se hace sistemáticamente. Por eso dicha herramienta se abandonó en el curso. 


\section{Conclusiones}

Este artículo presenta reflexiones sobre puntos que se consideran claves para la enseñanza-aprendizaje del $\mathrm{D}+\mathrm{E}$. Primero, muestra el $\mathrm{D}+\mathrm{E}$ dentro del marco conceptual del diseño para la afectividad, y más ampliamente, dentro del diseño para la UX. Segundo, presenta una serie de conceptos que sirven a fundamentar el curso. Estos provienen de teorías válidas y robustas sobre las emociones y que han sido adaptadas al diseño. El artículo presenta una selección de herramientas para el $\mathrm{D}+\mathrm{E}$, desde heurísticos para diseñar para el apego, hasta la KE, así como una discusión sobre sus condiciones de aplicación. Se piensa que estas herramientas, al estar basadas sobre hallazgos robustos, deberían poderse adaptar sin mayor problema en otros países de América Latina. Al día de hoy se tiene conocimiento de la existencia de cursos de D+E en México, Chile, Brasil y Colombia.

Con el curso aquí discutido se pretende cumplir con unos objetivos específicos. El primero es el de evidenciar y comprender cómo el D+E es un factor determinante para la definición de estrategias de diseño, desarrollo de productos y servicios innovadores. Se busca mostrar los beneficios del $\mathrm{D}+\mathrm{E}$ como un factor fundamental para la competitividad en un mercado globalizado, ya que se trata de un conjunto de técnicas de relativa fácil implementación y de rápidos resultados. Esta estrategia se opondría a un enfoque technology push que, al buscar innovar con tecnología, implica grandes inversiones, riesgos altos y resultados en el mediano o largo plazo. El segundo es el comprender cuáles son las dificultades inherentes al D+E y cómo sobrepasarlas. El tercero, es el de brindar modelos simples de cómo las personas sienten las emociones para así poder actuar sobre cada elemento que intervenga en dicho proceso. Por último se busca presentar y aplicar herramientas de D+E que tengan en cuenta, a través de su nivel de estructuración, las diferencias de recursos empresariales para el desarrollo de nuevos productos y servicios.

Este curso está orientado al diseño como disciplina que crea artefactos que prestan servicios a las personas. Es una disciplina orientada al hacer; por eso el énfasis del curso está en los métodos. La presentación de elementos teóricos tiene una doble finalidad: diferenciar las experiencias emocionales y sus componentes en la experiencia propia de los alumnos para que ellos sepan identificar y diferenciar, en ellos y en los demás, las emociones para 
las cuales diseñan y garantizar el uso adecuado de los métodos. La evaluación del curso se da entonces en dos niveles: los conceptos básicos del D+E y los resultados de la aplicación de los métodos, al hacer un uso mínimo y claro de los conceptos presentados en el curso cuando explican sus propuestas de diseño. Estos conceptos les deben servir para identificar el mecanismo que hay detrás de un diseño para las emociones propuesto. Al enfocarnos sobre los métodos nos interesa examinar si el método está entregando su promesa, es decir, por ejemplo, ¿el método de diseño para el wow presentado permite concebir un producto que genere wow en el usuario? No obstante, tratándose de métodos heurísticos, es difícil exigir unos resultados estrictos en este sentido.

El curso se presenta dentro de la filosofía de diseño integrado del producto, en la que para llegar a un producto exitoso, el producto que se está diseñando se ataca desde todos los frentes posibles dentro del tiempo y recursos que se tengan. Es por esto que se exige que de cada uno de los grupos de métodos se presente una alternativa y que luego, basándose en éstas, se presente una propuesta final que mejore significativamente la relación emocional con el producto.

Por último, una de las intenciones más importantes del artículo es la de compartir los resultados del montaje, desarrollo y evaluación de un curso de D+E para que sirva para establecer currículos similares en otras instituciones educativas. Se trata de un antecedente que puede interesar y orientar tanto a investigadores del tema como a profesores de diseño y que puede advertir sobre los escollos en el camino y sobre los fundamentos sobre los cuales apuntalar los contenidos de un curso de esta naturaleza. 


\section{Referencias}

Abbasi, M. Q., Weng, J., Wang, Y., Rafique, I., Wang, X. \& Lew, P. (2012). Modeling and Evaluating User Interface Aesthetics Employing ISO 25010 Quality Standard. Quality of Information and Communications Technology (QUATIC), 2012 Eighth International Conference, 303-306.

Ariza, N. \& Maya, J. (2014). Towards An Empirical Model of the UX: A Factor Analysis Study. En: Salamanca, J., Desmet, P., Burbano, A., Ludden, G., Maya, J. (Eds.), Proceedings of the 9th International Conference on Design and Emotion: The Colors of Care. 689-697.

Barrett, L. F. \& Rusell, J. A. (2009). Circumplex Models. En: The Oxford Companion to Emotion and the Affective Sciences.

Blythe, M. A., Overbeeke, K., Monk, A. F. \& Wright, P. C. (2004). Funology: from usability to enjoyment (Vol. 3). Springer.

Bridgens, B., Lilley, D., Smalley, G. \& Balasundaram, K. (2015). Ageing gracefully to increase product longevity. Proceedings: Product Lifetimes and the Environment, 19.

Byom, L. J. \& Mutlu, B. (2013). Theory of mind: Mechanisms, methods, and new directions. Frontiers in human neuroscience, 7. Frontiers Media SA.

Cadavid, Ana., Ruiz-Córdoba, Stefany \& Maya, Jorge. (2016). Extracting Design Aesthetic Heuristics from Scientific Literature.

Calle-Escobar, M., Mejia-Gutierrez, R., Nadeau, J.-P. \& Pailhes, J. (2014). Heuristics-based design process. International Journal on Interactive Design and Manufacturing (IIIDeM), 1-18. Springer.

Calle-Escobar, M., Mejia-Gutiérrez, R. \& Pailhes, J.-P. N. J. (2014). Methodology for the implementation of heuristics in the design process.

Chapman, J. (2009). Design for (emotional) durability. Design Issues, 25(4), 29-35. MIT Press.
Engage Consortium. (2008.). Tools to design for emotion. Recuperado de: http://www. desingandemotion.org/engage/sharmg_tools_a

Daly, S. R., Christian, J. L., Seifert, C. M., Gonzalez, R. \& Yilmaz, S. (2014). Design Heuristics 77 Cards. Design Heuristics, LLC.

Darbyshire, P., Bell, R. \& McDonald, H. (2006). Testing the circumplex model of emotions in a consumer setting. ANZMAC 2006: Advancing theory, maintaining relevance, proceedings.

Demir, E., Desmet, P. \& Hekkert, P. (2009). Appraisal patterns of emotions in human-product interaction. International Journal of Design, 3(2), 41-51.

Desmet, P., van Erp, J. \& Karlsson, M. (2008). Design \& emotion moves. Cambridge Scholars Publishing.

Desmet, P. M. (2002). Designing emotion. TU Delft Ph.D. Thesis.

Desmet, P. M. \& Hekkert, P. (2007). Framework of product experience. International Journal of Design, $1(1), 57-66$.

Desmet, P. M., Porcelijn, R. \& Van Dijk, M. (2007). Emotional Design; Application of a research-based design approach. Knowledge, Technology \& Policy, 20(3), 141-155. Springer.

Eagleman, D. (2011). Incognito. The secret lives of the brain. Vintage Books.

Ericsson, K. A., Simon, H.A. (1993). Protocol analysis: verbal reports as data.

Revised Edition. Cambridge-Massachusetts/MA: MIT Press, $443 \mathrm{p}$.

Fujita, K. \& Matsuo, T. (2005). Utilization of product development tools and methods: Japanese survey and international comparison. ICED 05: 15th International Conference on Engineering Design: Engineering Design and the Global Economy, 1661. 
Gigerenzer, G. \& Todd, P. M. (1999). Simple heuristics that make us smart. Oxford University Press.

Grimaldi, S. (2008). The Ta-Da series. A technique for generating surprising designs based on opposites and gut reactions (chapter 8). En: Desmet, P., van Erp, J. \& Karlsson, M. (2008). Design \& emotion moves. Cambridge Scholars Publishing.

Hassenzahl, M. \& Tractinsky, N. (2006). User experience a research agenda. Behaviour \& Information Technology, 25(2), 91-97. Taylor \& Francis.

Hekkert, P. \& Leder, H. (2008). Product aesthetics. In Schifferstein, HNJ, Hekkert, P. (Ed.), Product experience, 259-285. Elsevier Science San Diego, CA.

Hekkert, P. (1995). Artful judgements: A psychological inquiry into aesthetic preference for visual patterns. TU Delft Ph.D. Thesis

Hekkert, P. \& Schifferstein, H. N. (2008). Introducing product experience. Product experience, 1-8.

Hekkert, P., Thurgood, C. \& Whitfield, T. (2013). The mere exposure effect for consumer products as a consequence of existing familiarity and controlled exposure. Acta psychologica, 144(2), 411-417. Elsevier.

Jones, B. A. (2014). ADDIE Model (Instructional Design). Citeseer.

Jordan, P. W. (1998). Human factors for pleasure in product use. Applied ergonomics, 29(1), 25-33. Elsevier.

Jordan, P. W. (2002). Designing pleasurable products: An introduction to the new human factors. CRC Press.

Karapanos, E. (2013). Modeling Users' Experiences with Interactive Systems. Springer.

Klcinc, S. S. \& Baker, S. M. (2004). An integrative review of material possession attachment. Academy of Marketing Science Review, 2004(1), 1-35.
Krippendorff, K. \& Butter, R. (1984). Product semantics: Exploring the symbolic qualities of form. Innovation, 3(2).

Law, E. L.-C., Vermeeren, A. P., Hassenzahl, M. \& Blythe, M. (2007). Towards a UX manifesto. Proceedings of the 21st British HCl Group Annual Conference on People and Computers: HCl... but not as we know it-Volume 2 (pp. 205-206).

Law, E., Vermeeren, A., Hassenzahl, M. \& Eds, M. B (2004). Towards a UX Manifesto COST294-MAUSE affiliated workshop.

Leder, H., Belke, B., Oeberst, A. \& Augustin, D. (2004) A model of aesthetic appreciation and aesthetic judgments. British journal of psychology, 95(4), 489-508. Wiley Online Library.

Lokman, A. M. (2010). Design \& Emotion: The Kansei Engineering Methodology. Design \& Emotion: The Kansei Engineering Methodology, 1(1).

Ludden, Gekke (2008). Sensory Incongruity and surprise in product design. PhD Thesis, TU Delft.

Matzler, K. \& Hinterhuber, H. H. (1998). How to make product development projects more successful by integrating Kano's model of customer satisfaction into quality function deployment. Technovation, 18(1), 25-38. Elsevier.

Maya, J. (2010). Contribution à l'aide Au Choix des Méthodes d'Intégration de la Subjectivité de l'usager en Conception de Produits. Thèse Ph.D. Universidad de Tecnología de Compiègne. Compiègne, France.

Marina, José Antonio (2015). "Talento: inteligencia en acción". (1:31 a 1:45) Conferencia pronunciada en la sede de la Fundación Barrié en el marco de la Jornada "Educar hoy en contextos diferenciados". Recuperado de: https://www.youtube.com/ watch?v=WR2BNY5IIGA

Merrill, D., Drake, L., Lacy, M., Pratt, J. (1996). Reclaiming Instructional Design. Educational Technology, 36 (5), 5-7 
Mugge, R., Schifferstein, H. N. \& Schoormans, J.

P. (2010). Product attachment and satisfaction: understanding consumers' post-purchase behavior. Journal of Consumer Marketing, 27(3), 271-282. Emerald Group Publishing Limited.

Mugge, R., Schoormans, J. P. \& Schifferstein, H. N (2008). Product attachment: Design strategies to stimulate the emotional bonding to products. Product experience, 425-440. Elsevier Amsterdam.

Myers, D. G. (2004). Intuition: Its powers and perils. Yale University Press.

Nagamachi, M. (1995). Kansei engineering: a new ergonomic consumer-oriented technology for product development. International Journal of industrial ergonomics, 15(1), 3-11. Elsevier.

Nagamachi, M. (2002). Kansei engineering as a powerful consumer-oriented technology for product development. Applied ergonomics, 33(3), 289-294. Elsevier.

Nagamachi, M. (2010). Kanseilaffective engineering. CRC Press.

Nagamachi, M. \& Lokman, A. M. (2010). Innovations of Kansei engineering. CRC Press.

Norman, D. A. (2007). Emotional design: Why we love (or hate) everyday things. Basic books.

Ortíz-Nicolás, J. C. \& Aurisicchio, M. (2011). A scenario of user experience. Proceedings of the 18th International Conference on Engineering Design (ICED11), Vol. 7 (pp. 182-193).

Ortíz-Nicolás, J. C. \& López, I. H. (2008). Product relevant emotions in the Spanish language. Proceedings of The 6th International Conference on Design and Emotion.

Osgood, C. E. (1962). Studies on the generality of affective meaning systems. American Psychologist, 17(1), 10. American Psychological Association.
Osgood, C. E. \& Suci, G. J. (1955). Factor analysis of meaning. Journal of experimental psychology, 50(5), 325. American Psychological Association.

Plutchik, R. (2003). Emotions and life: Perspectives from psychology, biology, and evolution. American Psychological Association.

Posner, J., Russell, J. A. \& Peterson, B. S. (2005) The circumplex model of affect: An integrative approach to affective neuroscience, cognitive development, and psychopathology. Development and psychopathology, 17(03), 715-734. Cambridge Univ Press.

Van Rompay, T., Hekkert, P., Saakes, D. \& Russo, B. (2005). Grounding abstract object characteristics in embodied interactions. Acta psychologica, 119(3), 315-351. Elsevier.

Roozenburg, N. F. \& Eekels, J. (1995). Product design: fundamentals and methods (Vol. 2). Wiley Chichester.

Russell, J. A. (1980). A circumplex model of affect. Journal of personality and social psychology, 39(6), 1161. American Psychological Association.

Sander, D. \& Scherer, K. (2009). Oxford companion to emotion and the affective sciences. Oxford University Press.

Scherer, K. R. (2005). What are emotions? And how can they be measured? Social science information, 44(4), 695-729. Sage Publications.

Schifferstein, H. N. \& Hekkert, P. (2008). Product experience. Elsevier.

Schifferstein, H. N. \& Zwartkruis-Pelgrim, E. P. (2008). Consumer-product attachment: Measurement and design implications. International Journal of Design, 2(3), 1-13. Taylor \& Francis.

Schütte, S. T., Eklund, J., Axelsson, J. R. \& Nagamachi, M. (2004). Concepts, methods and tools in Kansei Engineering. Theoretical Issues in Ergonomics Science, 5(3), 214-231. Taylor \& Francis. 
Schwarz, N. (1999). Self-reports: how the questions shape the answers. American psychologist, 54(2), 93. American Psychological Association.

Schwitzgebel, E., 2016. Introspection. In Zalta, Edward N., (Eds.): The Stanford Encyclopedia of Philosophy. Metaphysics Research Lab, Stanford University. Available at: https://plato.stanford.edu/archives/ win2016/entries/introspection/ [Accessed 26-12, 2016].

Tonetto, L. \& Tamminen, P. (2015). Understanding the role of intuition in decision-making when designing for experiences: contributions from cognitive psychology. Theoretical Issues in Ergonomics Science, 16(6), 631-642. Taylor \& Francis.

Van Gorp, T. \& Adams, E. (2012). Design for emotion. Elsevier.

Vermeeren, A. P., Law, E. L.-C., Roto, V., Obrist, M., Hoonhout, J. \& Väänänen-Vainio-Mattila, K. (2010). User experience evaluation methods: current state and development needs. Proceedings of the 6 th Nordic Conference on Human-Computer Interaction: Extending Boundaries (pp. 521-530).

Vogel, C. M. \& Cagan, J. (2001). Creating breakthrough products: Innovation from product planning to program approval. Ft Press.

Wilson, R. A. \& Keil, F. C. (2001). The MIT Encyclopedia of the Cognitive Sciences (New Ed.). The MIT Press.

Wright, I., Campello, A., Segre, F., Benedetto-Neto, H. \& Araujo, C. (1995). A survey of methods utilisation during product design process in UK industry. Engineering Design Institute, Loughborough University of Technology.

Yilmaz, S. (2010). Design Heuristics. Ph.D. Thesis, University of Michigan. 